\title{
Toward a design method for metal-composite co-cured joints based on the G-SIFs
}

\author{
A. Russo ${ }^{\text {a }}$, B. Zuccarello ${ }^{\text {b,* }}$ \\ a ALENIA Aermacchi SPA, Via Ing. P. Foresio, 1, 21040 Venegono Superiore, VA, Italy \\ ${ }^{\mathrm{b}}$ Università di Palermo, Dipartimento di Ingegneria Chimica, Gestionale, Informatica, Meccanica Viale delle Scienze, 90128 Palermo, Italy
}

\section{A R T I C L E I N F O}

\section{Article history:}

Received 1 March 2012

Received in revised form 28 August 2012

Accepted 31 August 2012

Available online $\mathrm{xxxx}$

\section{Keywords:}

A. Polymer-matrix composites (PMCs)

B. Strength

C. Analytical modeling

E. Joints/joining

\begin{abstract}
A B S T R A C T
In this work, a systematic study of the singular stress field in the zone where the interface intersects the free edge surfaces of bonded metal-composite co-cured joints, has been performed. The obtained theoretical, numerical and experimental results have permitted to detect the relationships between the joint configuration and the singular stress field, as well as to implement a new design method based on the so called generalised stress intensity factors. Such a proposed method allows the user to predict the static strength of a generic metal-composite co-cured joint, vs. the main influence parameters as the elastic modulus of the coupled materials, the overlap length, the taper angle, the imbalance, etc. The accuracy of the theoretical and numerical analyses performed in the present study, as well as the reliability of the strength predictions provided by the proposed method, have been tested by means of experimental assessments carried out by using aluminum-GFRP and aluminum-CFRP co-cured double lap joints.
\end{abstract}

(c) 2012 Elsevier Ltd. All rights reserved.

\section{Introduction}

Thanks to the high mechanical strength, as well as to the low specific weight and the high corrosion resistance, Polimer Matrix Composites (PMCs) are widely employed in the mechanical design of modern high-performance lightweight structures, i.e. structures characterized by high strength and low weight (aeronautical and aerospace industries) or characterized by high corrosion resistance and low cost (chemical industry, petroleum or gas industry, etc.).

Recently, thanks also to their simple and economic manufacturing processes, PMCs are increasingly used in the civil engineering field for the repairing and for the reinforcement of structures made by masonry or concrete.

In all these structural applications, a special attention should be given to the design of the junctions used to assembly the various parts of the same component, as well as to join the reinforcement elements to the damaged structure (as in the structural reinforcement) or to connect components realized by using different materials, as in the common case in which modern structural components made by PMCs are joined to the main structure made by traditional materials.

Although these joints can be carried out by using traditional techniques based on rivets or bolts [1,2], if one or both the components to be joined are made by PMC, then they are usually joined by using an adhesively bonded joint or a so called bonded co-cured joint (or simply co-cured joint) in which the adhesive function is

\footnotetext{
* Corresponding author.

E-mail addresses: andrea.russo@alenia.it (A. Russo), bernardo.zuccarello@ unipa.it (B. Zuccarello).
}

performed by the matrix of the PMC that constitutes one of the adherents. In the last years these co-cured joints are increasingly used for the construction of advanced structures since, in addition to the advantages of classical adhesively bonded joints, they offer other particular advantages and are also characterized by a simpler manufacturing process because unlike the classical adhesively bonded joint, the surface treatments of the composite adherent are not necessary.

Unfortunately, nowadays the designer does not own reliable theoretical methods for the strength prediction of such joints and, consequently, their mechanical design is strictly related to unavoidable and often complex experimental assessments [3-9]. In fact, the absence of a proper adhesive layer does not allows the designer to use the classical theoretical methods [10] implemented for the adhesively bonded joints.

The first theoretical analysis of the stress singularity that characterizes the zone where the interface of a bi-material joint intersects the free edge, has been carried out in the 1950s by Williams [11] that considered the simple case of two isotropic adherents; successively, by using the Lekhnitski theory [13], Suo [12] has proposed a method for the theoretical analysis of the singularity orders of the stress field in case of adherents made by orthotropic materials. More recently, by systematic experimental analyses Tan [14] and Zuccarello [15] have implemented experimental methods, based on digital photoelasticity that allows the user the experimental evaluation of the Generalised Stress Intensity Factors (G-SIFs).

In order to implement a design method for the prediction of the static strength of hybrid metal-composite co-cured joints, based on $\mathrm{G}$-SIFs, in the present work a systematic theoretical and numerical 
study of double lap co-cured joints, followed by experimental tests, has been performed.

\section{Theoretical analysis}

The theoretical analysis has been performed by considering double lap joints in the general case in which both the adherents are made by anisotropic materials. Fig. 1 shows the schematic model of the co-cured double lap joint considered, and the relative geometrical parameters (thickness, overlap length, taper angle).

Taking into account that in practical applications the width of the joint is generally very large with respect to the thickness of the adherents, the theoretical analysis has been carried out by assuming a Plane Strain State (PSS). The solution of the elastic problem related to such a joint, can be achieved by using the Lekhnitskii theory [13,16-19], based on the so called complex potential method.

In particular, by considering a cylindrical reference system $(r, \theta, s)$ with origin coincident with the intersection between the joint interface and the free edge (see Fig. 1b), both the stress field and the displacements field in the singularity zone (close to the origin) can be expressed by means of functions with separated variables, that for each materials can be written as:

$$
\begin{aligned}
& \sigma_{i j}(r, \vartheta)=\sum_{v} K_{v} \cdot r^{-\omega_{v}} \cdot f_{i j}^{v}(\vartheta) \\
& u_{i}(r, \vartheta)=\sum_{v} K_{v} \cdot r^{1-\omega_{v}} h_{i}^{v}(\vartheta) \quad(i, j=r, \theta, s)
\end{aligned}
$$

In Eq. (1) the $K_{v}$ coefficient relative to the singular terms (i.e. to the terms having $\left.\omega_{v}>0\right)$ are the G-SIFs, $\omega_{v}$ are the singularity orders whereas $f_{i j}^{v}(\theta)$ and $h_{i}^{v}(\theta)$ are the angular functions of the stress field and of the displacement field, respectively; for their detailed expressions see Appendix A or Ref. [20].

By imposing to the stress equations provided by the complex potential method [13,16-19], the boundary conditions of the examined joint, as:

(a) Nullity of both normal and tangential stresses on the free edge surfaces $\left(\theta=0^{\circ}\right.$ and $\theta=\varphi$, see Fig. 1$)$;

(b) Equality of normal and the tangential stresses of the two coupled materials at the joint interface $\left(\theta=180^{\circ}\right)$;

then the following set of equations constituted by 12 homogeneous equations, is obtained:

$[M(\omega)] \cdot\left(\begin{array}{lllll}a_{11} & \bar{a}_{11} & \cdots & c_{12} & \bar{c}_{12}\end{array}\right)^{T}=\left(\begin{array}{lllll}0 & 0 & \cdots & 0 & 0\end{array}\right)^{T}$

As it is well known, this set of equations has a solution different from the banal one only if the matrix $[\mathrm{M}(\omega)]$ is singular, i.e. if:

$\operatorname{Det}[M(\omega)]=0$

The solution of Eq. (3) gives the stress singularity orders $\omega_{v}$ (eigenvalues). For each eigenvalue the successive solution of Eq. (2) permits to determine the corresponding angular function $h_{k, i}^{v}(\theta)$ and $f_{k j, i}^{v}(\theta)$ from the constants $a_{1, i}, a_{2, i}, b_{1, i}, b_{2, i}, c_{1, i}, c_{2, i}$ (eigenvectors). In general Eq. (3) has one or more solutions that can be complex or real. As it is well known from the typical eigenvalues-eigenvectors problem, in any case each solution is defined univocally, except for an unknown constant. Therefore, to overcome such a mathematical problem, the unitary value is imposed to the angular functions $f_{\theta \theta}^{v}$ at the interface $\left(\theta=180^{\circ}\right)$, i.e. $f_{\theta \theta, 1}^{v}\left(180^{\circ}\right)=f_{\theta \theta, 2}^{v}\left(180^{\circ}\right)=1$; from these conditions it follows:

$\sigma_{\vartheta \vartheta, i}\left(r, 180^{\circ}\right)=\sum_{v} K_{v} \cdot r^{-\omega_{v}}$

By means of the above exposed approach properly developed in more detail by Chue et al. in [16-19], 9 different double lap metal-composite co-cured joints have been analyzed; they are obtained by considering all the combinations between three different metals (steel, aluminum, titanium) and three different unidirectional composites as Glass Fiber Reinforced Plastic (GFRP), Aramid Fiber Reinforced Plastic (AFRP) and Carbon Fiber Reinforced Plastic (CFRP), whose elastic characteristics are reported in Table 1 . In practice, such combinations permit to cover the range in which the joints, actually used in aeronautical/aerospace and nautical field, fall.

The above exposed theoretical approach has shown that such joints are in general characterized by two singularity orders in the plane $x y$ (in-plane singularities $\omega_{1, i}$ and $\omega_{2, i}, i=A, B$ ) and one singularity order out of plane $x y$ (anti-plane singularity $\omega_{3, i}, i=A$, B) $[16,21]$.

As an example, Fig. 2 shows the singularity orders in the points A (Fig. 2a) and B (Fig. 2b) of an aluminum-AFRP joint, vs. the taper angle $\varphi$ of the composite adherent. In particular, from Fig. 2a it is possible to observe how in the taper angle range of practical interest $\left(90^{\circ} \leqslant \varphi \leqslant 180^{\circ}\right)$, in the plane $x y$ the singular stress field is described in practice by one singularity $\left(\omega_{1, A}\right)$, being $\omega_{2, A} \approx 0$ for $\varphi \geqslant 90^{\circ}$. Also, Fig. 2a shows that the in-plane and the anti-plane singularity orders decrease when $\varphi$ increases; in detail, as it is expected, they tend to zero when $\varphi$ tends to $180^{\circ}$.

On the contrary, Fig. 2b shows that for point B the singularity zone is governed by two in-plane singularity orders $\left(\omega_{1, B}, \omega_{2, B}\right)$ and one anti-plane singularity order $\left(\omega_{3, B}\right)$.

Moreover, by comparing the results relative to points $\mathrm{A}$ and $\mathrm{B}$, it is seen that for $90^{\circ} \leqslant \varphi \leqslant 180^{\circ}$ the singularity orders of the point $B$ are always higher than those relative to the point $A$.

In the following sections the "out of plane" singularity orders $\left(\omega_{3, A}, \omega_{3, B}\right)$ will not be considered anymore because the corresponding SIFs (due to the coupling between the in plane and the out of plane stresses) are always negligible for joints between anisotropic materials [22]; in other words, it means that for these joints the in plane and the out of plane stress states can be considered in practice uncoupled.

In order to highlight the influence of the materials on the singularity order values, Fig. 3 shows the $\omega_{1, A}, \omega_{1, B}$ and $\omega_{3, B}$ singularity

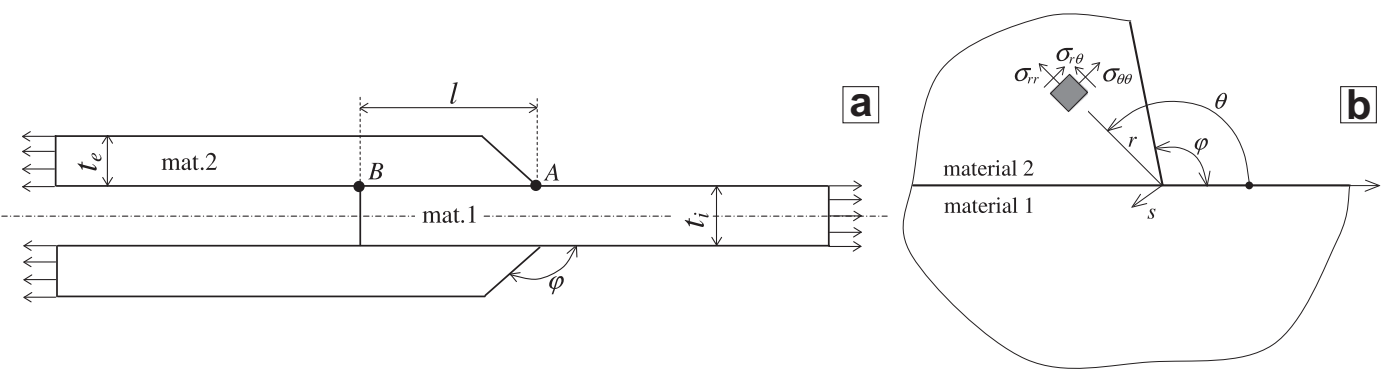

Fig. 1. (a) Geometry of the examined double lap co-cured joint and (b) cylindrical reference system. 
Table 1

Properties of the materials considered in the theoretical and numerical analyses.

\begin{tabular}{lrlll}
\hline Material & $E_{L}(\mathrm{GPa})$ & $E_{T}, E_{Z}(\mathrm{GPa})$ & $G_{L T}(\mathrm{GPa})$ & $v_{L T}$ \\
\hline Steel & 210.0 & & & 0.33 \\
Titanium & 114.0 & & & 0.33 \\
Aluminum & 72.0 & & & 0.29 \\
CFRP & 121.5 & 6.2 & 2.2 & 0.29 \\
AFRP & 67.1 & 6.1 & 2.2 & 0.37 \\
GFRP & 44.1 & 6.0 & 2.1 & 0.30 \\
\hline
\end{tabular}

orders for different composite materials coupled with aluminum (internal adherent, Fig. 3a), as well as for different metals coupled with GFRP (external adherent, Fig. 3b).

From Fig. 3a, it is seen that if the metal of the internal adherent is fixed, then in the point $\mathrm{A}$ (attach point of the metallic adherent) the singularity order $\omega_{1 A}$ increases with the elastic modulus of the composite materials, i.e. by passing from the GFRP to the AFRP to the CFRP. An opposite behavior is exhibited by $\omega_{2 B}$ (point B), whereas $\omega_{1 B}$ remains quite constant for any elastic modulus value of the external adherent in the examined range.

On the contrary, from Fig. $3 \mathrm{~b}$ it is seen that if the composite material of the external adherents is fixed, than in the point $A$ the singularity order increases when the elastic modulus of the internal metallic adherent decreases, i.e. by passing from steel to titanium to aluminum. Significant variations of the singularity orders occur also in the point B but only for $\omega_{2 B}$ which, unlike $\omega_{1 A}$, increases with the elastic modulus of the metallic adherent.
Finally, it has to be noted that although the analyses carried out in the present work are restricted to unidirectional composite laminates, it does not constitute a limitation for the singularity order analyses because the optimal configuration (maximum strength) of a generic angle-ply laminates to be joined by using a co-cured joint (or a classical adhesively bonded joint) is always that in which the interface lamina is unidirectional with the fiber axis aligned with the joint axis.

\section{Numerical analysis}

The above reported theoretical analysis of the stress field around points A and B has shown that, for the values of the taper angle of practical interest, the singular stress components are described respectively by a monomial and a binomial relationship [11,23], i.e.:

$$
\begin{aligned}
& \sigma_{i j}(r, \vartheta)=K_{1, A} \cdot r^{-\omega_{1}} \cdot f_{i j, 1}(\vartheta) \quad(\text { point A) } \\
& \sigma_{i j}(r, \vartheta)=K_{1, B} \cdot r^{-\omega_{1}} \cdot f_{i j, 1}(\vartheta)+K_{2, B} \cdot r^{-\omega_{2}} \cdot f_{i j, 2}(\vartheta) \quad \text { (point B) }
\end{aligned}
$$

Therefore, like Linear Elastic Fracture Mechanics (LEFM), once the singularity orders and the angular functions are computed theoretically, then the corresponding in-plane G-SIFs $K_{1 A}, K_{1 B}$ and $K_{2 B}$, can be computed from the corresponding stress fields close to points $A$ and $\mathrm{B}$, provided by numerical simulations or experimental analyses.

In order to evaluate the G-SIFs relative to the singular points $\mathrm{A}$ and $B$, numerical simulations have been performed by using the Boundary Element Method (BEM) and the BEASY ${ }^{\circledR}$ code. In more

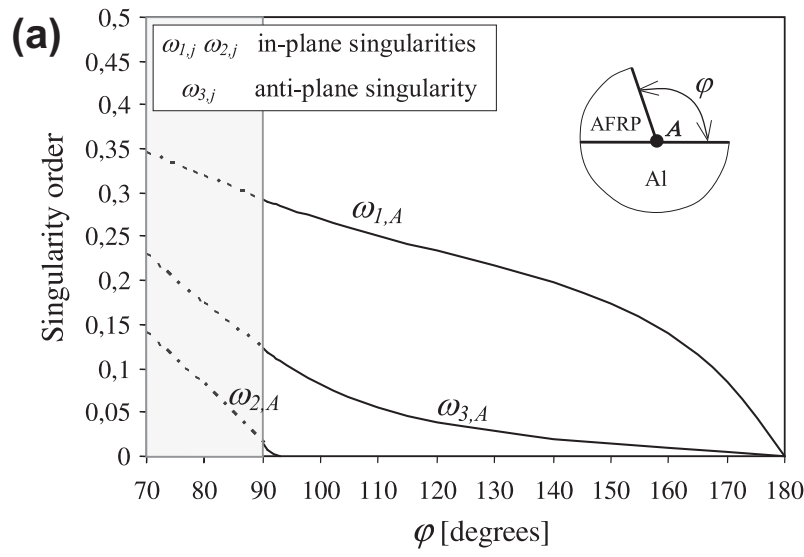

(b)

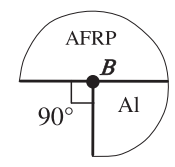

$\omega_{1, B}=0.499$

$\omega_{2, B}=0.316$

$\omega_{3, B}=0.476$

Fig. 2. Singularity orders vs. taper angle $\varphi$ for aluminum-AFRP joint (a) at the attach point of the metallic adherent and (b) at the attach point of the composite adherent.
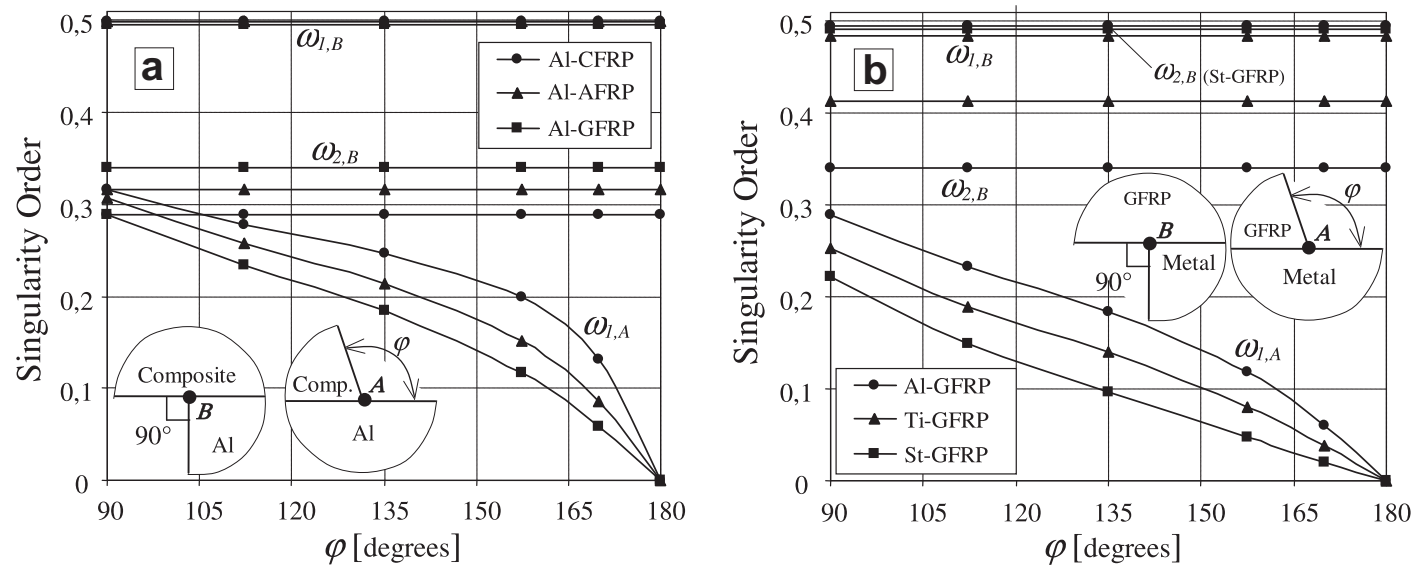

Fig. 3. Singularity orders vs. taper angle $\varphi$ for (a) aluminum-composite and (b) metal-GFRP joints. 


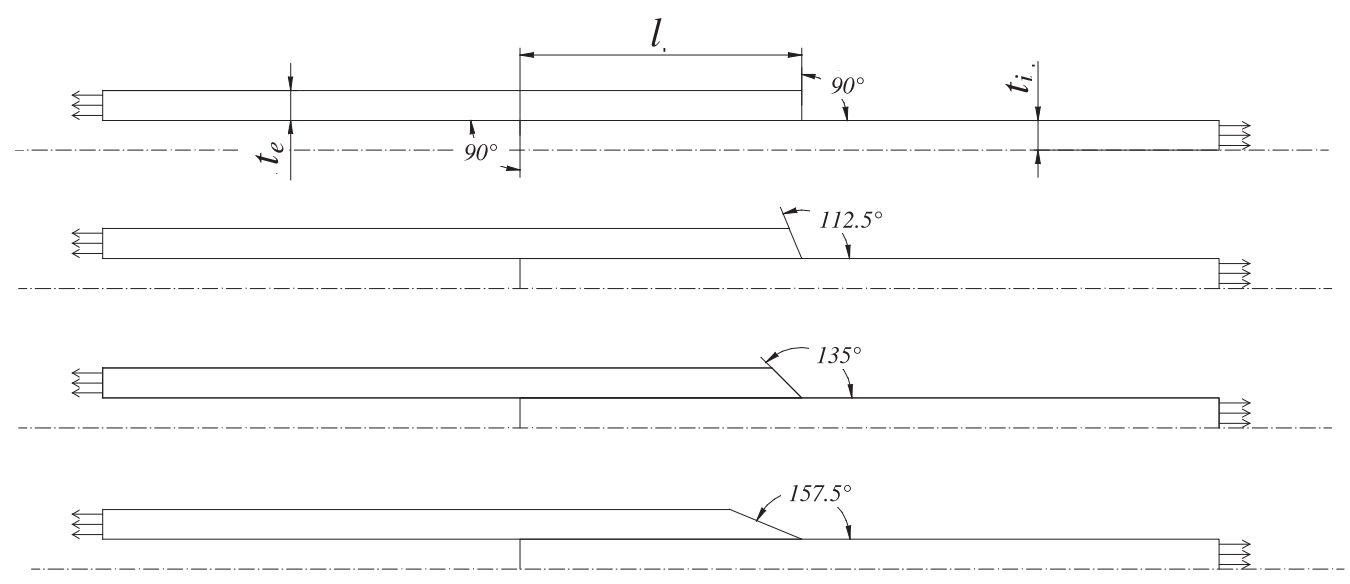

Fig. 4. Geometry of the double lap joints analyzed by using BEM.

detail, by using four different models corresponding to four different taper angles $\left(\varphi=90^{\circ}, 112.5^{\circ}, 135^{\circ}, 157.5^{\circ}\right.$ - see Fig. 4$)$, all the joints considered in the previous theoretical study, have been simulated numerically. Taking into account the symmetry of the joint with respect to the middle plane, each numerical model represents only half of the joint (see Fig. 4). Moreover, the models have been discretized by means of about 1600 quadratic elements with a progressive mesh grading close to the singular points A and B; in such a way the smaller elements have dimensions of about $1 \mu \mathrm{m}$ (see Fig. 5a).

For each considered joint, after the estimation of the extension of the Singularity Dominated Zone (SDZ), the G-SIFs have been computed by fitting the stress components provided numerically (Beasy $^{\circledR}$ code) by means of functions (5) and (6). As an example, Fig. 5b shows the distribution of the stresses $\sigma_{x}, \sigma_{y}$ and $\tau_{x y}$ close to the point $\mathrm{A}$, for an aluminum-AFRP joint having metallic adherent thickness $t_{i}=6 \mathrm{~mm}$, composite adherent thickness $t_{e}=3 \mathrm{~mm}$, overlap length $l=30 \mathrm{~mm}$, taper angle $\varphi=157.5^{\circ}$ and remote tensile stress $\sigma_{o}=50 \mathrm{MPa}$. Taking into account that in the SDZ the stresses are described by the exponential function (5), in the logarithm scale the extension of the SDZ corresponds with the rectilinear segment of the stress curves; in fact the logarithmic expression of Eq. (5) is a simple linear function as:

$\log \left[\sigma_{i j}(r, \vartheta)\right]=-\omega_{1} \log [r]+\log \left[K_{1, A} \cdot f_{i j, 1}(\vartheta)\right]$

This expression, as well as the graphics of the Cartesian stress components at the interface, suggests the user that the GSIF $K_{1, A}$ coincides with the inverse logarithm of the constant that appears into
Eq. (7) written for the stress $\sigma_{y}$ orthogonal at the interface (remember that $f_{y y}\left(180^{\circ}\right)=1$ at the interface).

This simple and reliable approach [24-26] has been used for the evaluation of G-SIFs by varying the main influence parameters [27-32] as the taper angle $\varphi$, the elastic constants of the adherents, the overlap/thickness ratio $l / t_{i}$, the relative thickness of the adherents $t_{i} / t_{e}$.

A similar approach $[15,20]$ based on the use of the polynomial Eq. (6), is used in the following sections to evaluate the two G-SIFs relative to the point $B$.

Finally, it is possible to observe that like LEFM, each GSIF can be expressed [33-35] as:

$K_{i j}=\beta_{i j} \sigma_{o} w^{-\omega_{i j}} \quad(i=1,2 ; j=A, B)$

where $\sigma_{o}$ is the applied remote stress, $\beta_{i j}$ are the shape functions (called also non-dimensional G-SIFs) that are related to the geometry and to the loading configuration, $w$ is a characteristic dimension of the joint, in the following assumed equal to the thickness $t_{i}$ of the metallic adherent.

In the following sections all the results are reported in terms of the non-dimensional parameters $\beta_{i j}$ because such a choice permits to represent in the same graph, results relative to different singularity orders, e.g. results relative to the points $\mathrm{A}$ and $\mathrm{B}$.

\subsection{Influence of the taper angle}

In order to detect the influence of the taper angle of the external adherents on the G-SIFs, Fig. 6a shows the non-dimensional G-SIFs

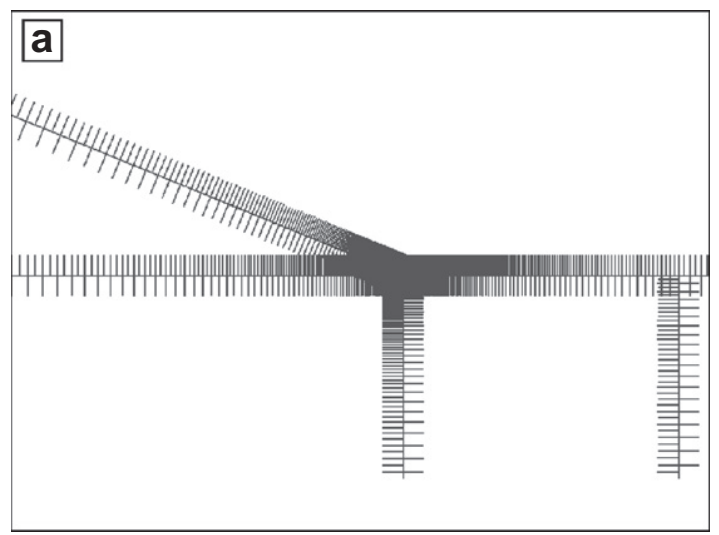

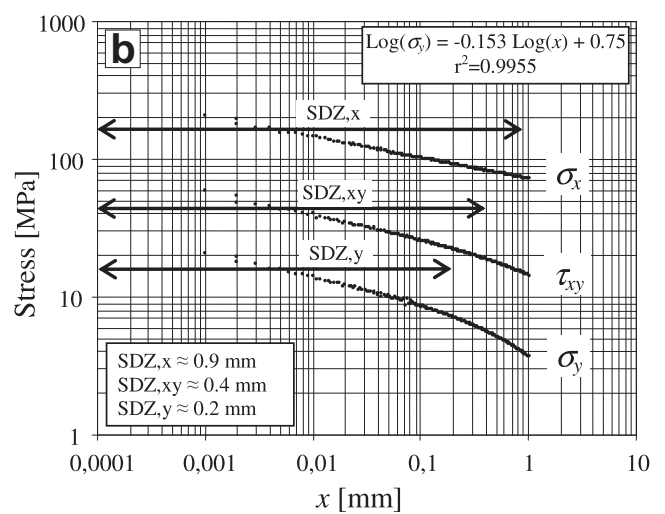

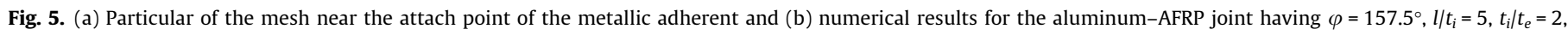
$\sigma_{o}=50 \mathrm{MPa}$. 

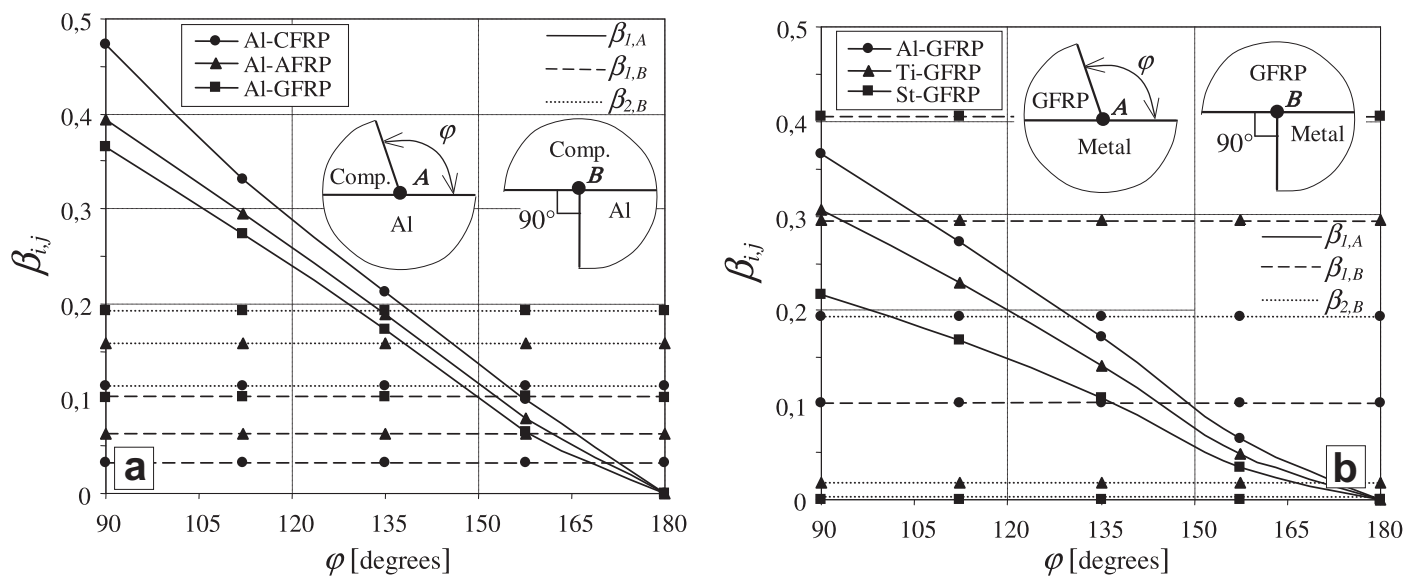

Fig. 6. G-SIFs vs. taper angle for (a) aluminum-composite joint having $t_{i} / t_{e}=3$ and $l / t_{i}=5$, (b) metal-GFRP joint having $t_{i} / t_{e}=3$ and $l / t_{i}=5$.

for aluminum-composite (GFRP, AFRP, CFRP) joints versus $\varphi$, for $t_{i} / t_{e}=3$ and $l / t_{i}=5$. It is possible to see that, like the singularity orders, $\beta_{1, A}$ tends to zero when $\varphi$ tends to $180^{\circ}$; also, for a fixed taper angle value, it increases with the longitudinal Young modulus of the composite adherent, i.e. by passing from GFRP to AFRP to CFRP. On the contrary, both $\beta_{1, B}$ and $\beta_{2, B}$ decrease when the longitudinal Young modulus of the composite increases. These results are in accordance with the experimental evidence as well as with the classical theory of the adhesively bonded joints, that shows how the most stressed zone passes from the point $B$ to the point $A$, increasing the external adherent stiffness; in other words the most stressed zone is always that one close to the attach point of the less stiff adherent.

In order to highlight also the effects of the internal adherent material (metal), Fig. 6b shows the curves of non-dimensional GSIFs $\beta_{i j}(i=1,2 ; j=A, B)$ for three different joints: aluminum-GFRP, titanium-GFRP and steel-GFRP. From this figure it is possible to observe how $\beta_{1, A}$ increases when the Young modulus of the metal decreases, i.e. by passing from steel to titanium to aluminum. On the contrary, $\beta_{1, B}$ (dominant GSIF) decreases with the metal stiffness, whereas $\beta_{2, B}$ increases.

Moreover, from Fig. 6 it is seen that in the singular zone close to point $\mathrm{A}$ the higher $\beta_{1, A}$ value corresponds to the non-tapered $\left(\varphi=90^{\circ}\right)$ aluminum-CFRP joint (that has the less stiff metallic adherent), whereas the lower $\beta_{1, A}$ value corresponds to the steelGFRP joint (that has the most stiff metallic adherent); on the contrary, in the singular zone close to point $\mathrm{B}$ the higher value of $\beta_{1, B}$ corresponds to the steel-GFRP joint (having the less stiff composite adherent) whereas the lower $\beta_{1, B}$ value corresponds to the aluminum-CFRP joint (having the most stiff composite adherent).

Also, it is important to observe that for a fixed joint, the $\beta_{1, A}(\varphi)$ curve intersects the $\beta_{1, B}(\varphi)$ and $\beta_{2, B}(\varphi)$ curves (see Fig. $6 a$ and $b$ ); consequently, by varying the taper angle the most stressed zone (failure zone) can pass from the attach point of the metallic adherent (point A) to the attach point of composite adherent (point B).

\subsection{Influence of the elastic modulus of the adherents}

In order to evaluate the influence of the mismatch of the elastic modules of the adherents on the G-SIFs [27-30], numerical simulations have been performed by varying the elastic properties of the adherents that constitute various co-cured joints with $t_{i} / t_{e}=3, l / t_{i}=5$ and $\varphi=90^{\circ}$.

From Fig. 7a it is possible to observe that near the attach point of the composite adherent (point $A$ ), the value of $\beta_{1, A}$ increases with the longitudinal elastic modulus of the composite adherent. Near the attach point of the metallic adherent (point B), instead, $\beta_{1, B}$ decreases, whereas $\beta_{2, B}$ takes a non-monotone trend; in more detail, $\beta_{2, B}$ is null for steel-GFRP and steel-AFRP that exhibits a single singularity (only $\beta_{1, B}$ is non-null).

The variations of $\beta_{1, B}$ can be better seen in Fig. $7 \mathrm{~b}$ that shows the non-dimensional G-SIFs versus the elastic modulus of the metallic adherent. It is seen that $\beta_{1, B}$ increases significantly with the elastic modulus of the metallic adherent, whereas both $\beta_{1, A}$ and $\beta_{2, B}$ exhibit a decreasing trend except $\beta_{2, B}$ for steel-CFRP joints.
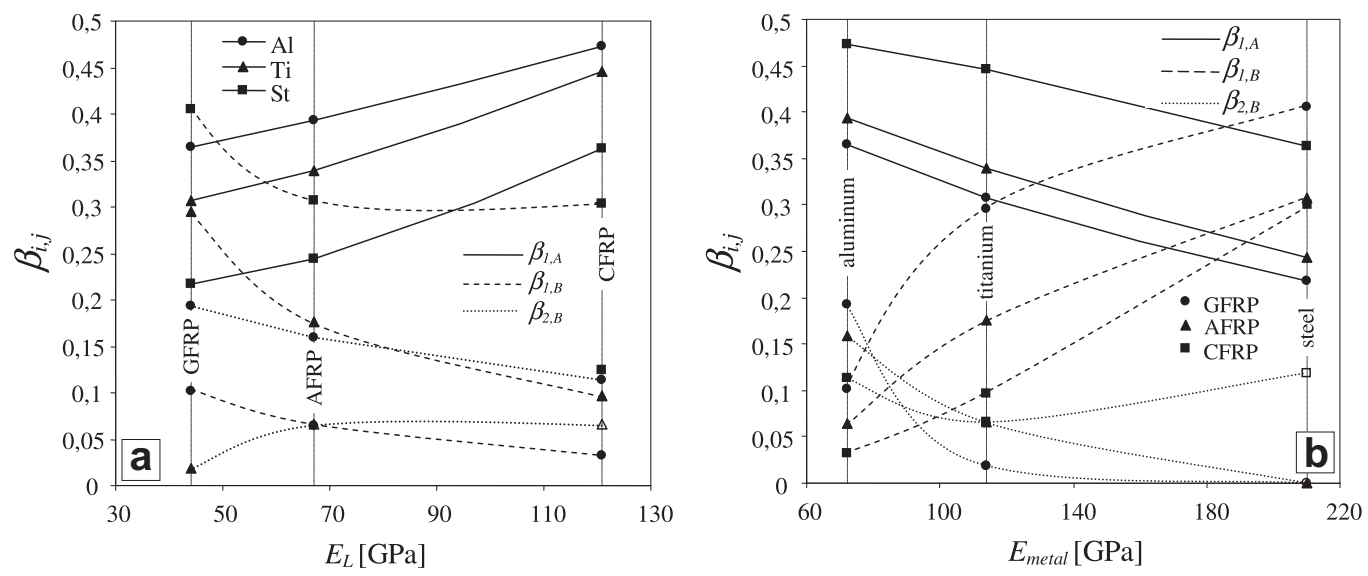

Fig. 7. G-SIFs vs. (a) the Young modulus $E_{L}$ of the composite and (b) the Young modulus of the metal. 


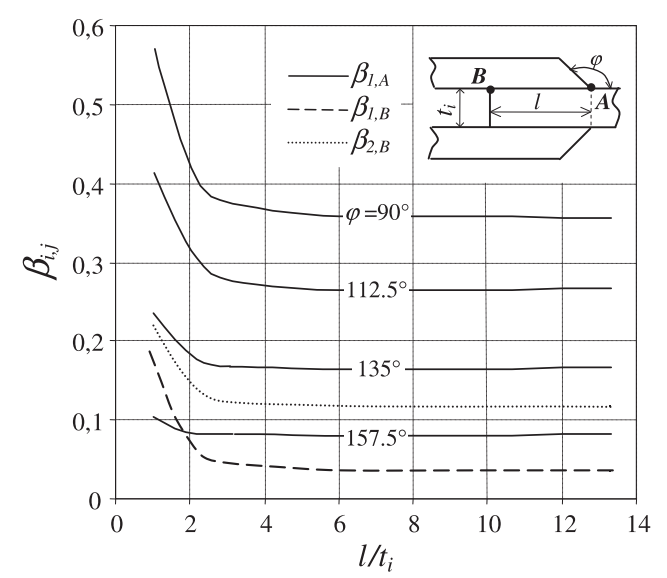

Fig. 8. G-SIFs vs. $l / t_{i}$ ratio for the aluminum-CFRP joint having $t_{i} / t_{e}=3.5$.

Finally, it is important to observe that for the point $\mathrm{B}$, the dominant non-dimensional G-SIF changes from $\beta_{2, B}$ to $\beta_{1, B}$ when $E_{\text {metal }}$ increases; therefore, for low values of $E_{\text {metal }}$ (e.g. $E_{\text {metal }} \leqslant E_{\text {aluminum }}$ ) it is possible to neglect $\beta_{1 B}$ with respect to $\beta_{2 B}$. On the contrary, for high values of $E_{\text {metal }}\left(\right.$ e.g. $E_{\text {metal }} \geqslant E_{\text {titanium }}$ ), it is possible to neglect $\beta_{2 B}$ with respect to $\beta_{1 B}$.

\subsection{Influence of the ratio $l / t_{i}$}

In order to evaluate the influence of the characteristic $l / t_{i}$ ratio between the overlap length and the internal adherent thickness, on the G-SIFs, numerical simulations of aluminum-CFRP joints (with $t_{i} / t_{e}=3.5$ ) have been performed by varying the $l / t_{i}$ ratio in a wide range.

Fig. 8 shows the curves of the non-dimensional G-SIFs relative to the points $A$ and $B$ versus the non-dimensional parameter $l / t_{i}$. From the analysis of such results it is seen that $\beta_{1, A}$ decreases when the parameter $l / t_{i}$ increases and tends to become constant for $l / t_{i}>6$. Qualitatively similar results are obtained for $\beta_{i, B}(i=1,2)$ as well as for joints with different coupled materials. Consequently, it is possible to state that for elastic materials all the G-SIFs decrease when $l / t_{i}$ increases until the value of about 6 ; above this value a further increasing of the overlap length does not lead to a further decreasing of the G-SIFs. Such results agree well with the independent experimental evidence [27-30] that has shown how the mechanical strength of an adhesively bonded joint increases with the overlap until a fixed value $\left(l / t_{i}\right)^{*}$, then it tends to become constant for $l / t_{i}>\left(l / t_{i}\right)^{*}$

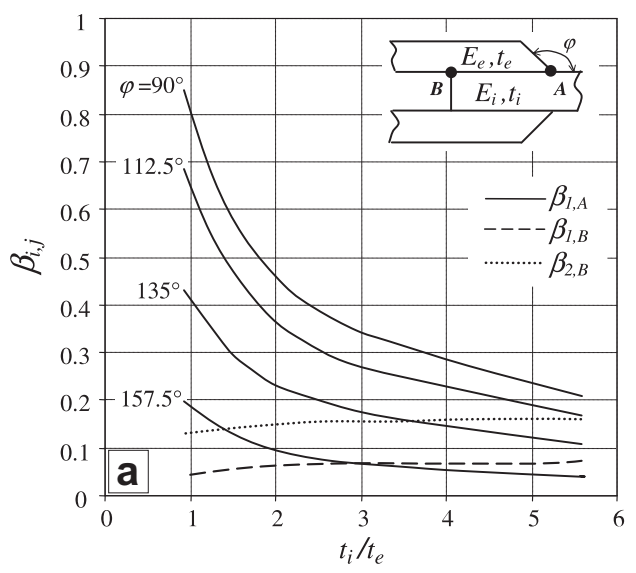

In other words it is possible to state that for $l / t_{i}>6$ in practice $\beta_{1, A} l / t_{i}, \varphi$ does not depend to $l / t_{i}$, but it depends only to the taper angle $\varphi$. In detail, in this range it takes the following simple expression, obtained by fitting the numerical results:

$\beta_{1, A}(\varphi)=\beta_{1, A}\left(90^{\circ}\right) \cdot\left(2-\varphi / 90^{\circ}\right)$ for $l / t_{i}>6$

Although Eq. (9) has been obtained for aluminum-CFRP joints, it can be applied also to the other examined joints. Obviously, Eq. (9) can be advantageously used at the design stage to compute the effects of the actual taper angle on the corresponding nondimensional SIF and, therefore, on the ultimate load (inversely proportional to the SIF).

\subsection{Influence of the adherents thickness}

In order to study the influence of the adherents thickness on the G-SIFs of a co-cured double lap joint [27-30,32], numerical simulations have been performed by varying the ratio $t_{i} / t_{e}$ for joints with $l / t_{i}=5$ and various values of $\varphi$. Fig. 9a shows the $\beta_{1, A}, \beta_{1, B}$ and $\beta_{2, B}$ curves for an aluminum-AFRP joint with various values of the taper angle, versus the ratio $t_{i} / t_{e}$. It is seen how $\beta_{1, A}$ decreases when $t_{i} / t_{e}$ increases, whereas $\beta_{1, B}$ and $\beta_{2, B}$ increase with $t_{i} / t_{e}$ although with very low variations. Also, it is possible to see that, if such curves are depicted in logarithmic coordinates (see Fig. 9b), then they became linear, with the same slope. In other words, for any $\varphi$ value, it is possible to write that $\operatorname{Ln}\left(\beta_{i, j}\right)=a_{i, j}+b_{i, j} \operatorname{Ln}\left(t_{i} / t_{e}\right)$, i.e.:

$\beta_{i, j}\left(t_{i} / t_{e}\right)=\beta_{i, j}(1) \cdot\left[t_{i} / t_{e}\right]^{b_{i, j}}$

For the considered aluminum-AFRP joints $b_{1, A} \approx 0.9, b_{1, B} \approx b_{2, B}$ $\approx 0.10$; such a formula is in general a useful tool for the designer; as an example it permits to compute quickly the SIFs when the thickness of one of the or both the adherents is properly varied near the overlap zone to increase the static strength of the joint. Qualitatively similar results have been obtained for different coupled materials.

Finally, from Fig. 9 it is important to observe how the curves of $\beta_{1, A}$ intersect those relative to $\beta_{1, B}$ and $\beta_{2, B}$, so that it is possible to state that when $t_{i} / t_{e}$ increases, the most stressed zone can pass from the attach point of the metallic adherent (point $A$ ) to the attach point of the composite adherent (point B).

\section{The proposed design method based on the G-SIFs}

As above observed, for a generic double lap tapered joint, the stress singularity varies with the taper angle $\varphi$ and, for a fixed value of $\varphi$, it is different for the two attach points of the adherents

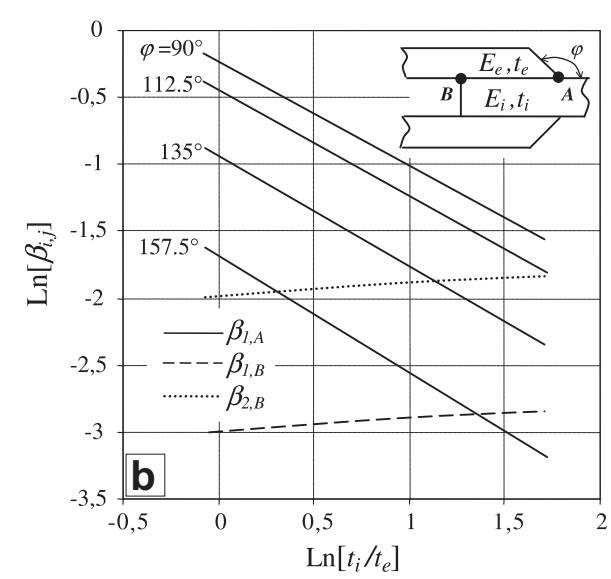

Fig. 9. G-SIFs of the aluminum-AFRP joint vs. $t_{i} / t_{e}$, represented by (a) linear coordinates and (b) by logarithmic coordinates. 
(points A and B). Also, in general near the attach point of the metallic adherent (point $A$ ) the stress singularity is governed by a unique singular term, whereas near the attach point of the composite adherent (point B) the stress singularity is described by two singular terms. Also, different singularity orders correspond to each singular term.

Therefore, unlike LEFM in which for any crack opening mode the corresponding strength criterion involves a unique SIF, for a generic hybrid double lap joint with adherents made also by anisotropic materials, a strength criterion based on the G-SIFs has to take into account the actual singularity orders that depend on the coupled materials and the taper angle. Also, for a fixed taper angle $\varphi$ of the composite adherents, and for a given couple of materials, the strength criterion must be applied separately to the two singular points A and B. Moreover, at the point B the strength criterion should involve both G-SIFs, by using an equivalent SIF $\beta_{\text {eq,B }}$ that, as an example, can be defined by the function $\beta_{\text {eq }, B}=\sqrt{\beta_{1, B}^{2}+\beta_{2, B}^{2}}$, widely proposed in literature for isotropic materials [33].

In order to detect the influence of each GSIF on the $\beta_{\text {eq,B }}$ defined by such a function, Fig. 10 shows the curves of $\beta_{B, e q}, \beta_{1, B}$ and $\beta_{2, B}$ versus $t_{i} / t_{e}$ for a double lap aluminum-GFRP co-cured joint. It is seen how $\beta_{2, B}$ is always higher than $\beta_{1, B}$ and the influence of $\beta_{1, B}$ can be neglected being the relative error $\Delta \%=100\left(\beta_{2, B}-\beta_{\text {eq }, B}\right) / \beta_{\text {eq, } B}$ always less than $8 \%$ (less than $5 \%$ in the usual condition in which $t_{i}$ | $t_{e} \geqslant 2$, see also Fig. 10). Similar results are obtained for the other coupled materials considered in the present study. Therefore, it is possible to state that for a generic hybrid double lap joint a strength criterion based on G-SIFs is in practice represented by a double strength condition as:

$K_{1, A} \leqslant K_{1, A-c}$

$K_{2, B} \leqslant K_{2, B-c}$

where $K_{1, A-c}$ and $K_{2, B-c}$ are the so called critical SIFs relative to the points $A$ and $B$ respectively.

From conditions $(11 \mathrm{a}, \mathrm{b})$, it is possible to evaluate the ultimate loads that corresponds to the joint failure with adhesive failure propagating from the point $A$ or $B$. In practice, by assuming $w=t_{i}$, for incipient failure that starts from the point A, Eq. (8) becomes:

$K_{1, A-c}=\beta_{1, A} \sigma_{u} t_{i}^{-\omega_{1, A}}$

whereas for incipient failure starting from the point $\mathrm{B}$, it becomes:

$K_{2, B-c}=\beta_{2, B} \sigma_{u} t_{i}^{-\omega_{2, B}}$

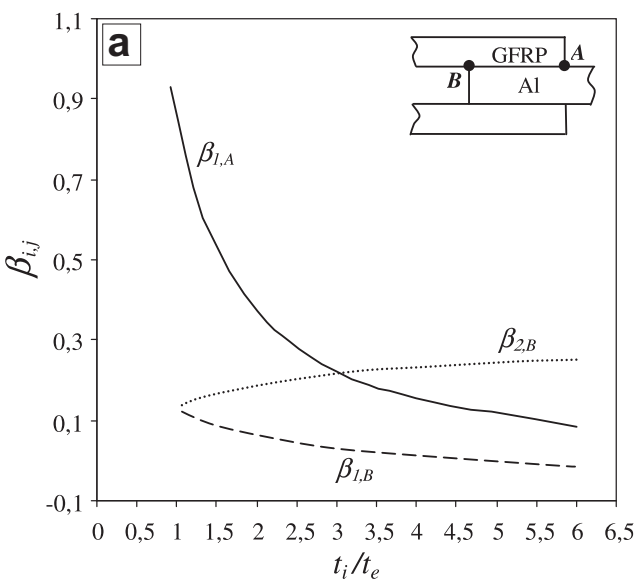

Into Eqs. $(12 \mathrm{a}, \mathrm{b}) \sigma_{u}$ is the nominal ultimate stress of the examined joint, achieved by dividing the failure load obtained experimentally, by the transversal section of the internal adherent. It is important to note that the evaluation of the critical SIFs performed by using Eqs. (12a,b) allows the user to take into account all the parameters that influence the actual bonding realized (roughness of the metal adherent surface, metal surface treatments, temperature and pressure of curing, environmental conditions, etc.).

After $\omega_{1, A}$ and $\omega_{2, B}$ are evaluated by the theoretical analysis (Eq. (3)), $\beta_{1, A}$ and $\beta_{2, B}$ are computed by numerical simulations or experimental analysis, as well as $\sigma_{u}$ is obtained from failure tests performed by using specimens similar to the examined joint (same configuration, materials and taper angle), then Eqs. (12a,b) permit in general the characterization of the joint by the evaluation of the corresponding critical SIF $K_{1, A-c}$ (adhesive failure starting from point A) or $K_{2, B-c}$ (adhesive failure starting from point B).

Also, at the design stage Eqs. (12) allows the user to compute the two specific (per unit width) ultimate loads $L_{u j}(j=A, B)$ of the examined joint by multiplying the ultimate stress by the internal adherent thickness; from Eqs. (12) it follows that:

$L_{u, A}=\sigma_{u} t_{i}=\frac{K_{1, A-c}}{\beta_{1, A}} \cdot t_{i}^{\left(1+\omega_{1, A}\right)}$

$L_{u, B}=\sigma_{u} t_{i}=\frac{K_{2, B-c}}{\beta_{2, B}} \cdot t_{i}^{\left(1+\omega_{2, B}\right)}$

Obviously, in terms of specific applied load $L_{\text {applied, }}$, the strength condition of the proposed method based on the G-SIFs is represented by the simple condition:

$L_{\text {applied }} \leqslant \min \left(L_{u, A}, L_{u, B}\right)$

\section{Experimental analysis}

In order to assess the theoretical and numerical results reported in the previous sections, as well as to test the proposed design method based on G-SIFs, various double lap aluminum-composite joints, have been subjected to experimental tests (Fig. 11). In detail, aluminum-GFRP and aluminum-CFRP joints have been tested in accordance with the ASTM D3528 standard [36].

All the examined joints have width of $25 \mathrm{~mm}$, taper angle $\varphi=0$, internal metallic adherent thickness $t_{i}=3 \mathrm{~mm}$, whereas the different composite adherent thicknesses correspond to $t_{i} / t_{e}=0.92,1.33$, $2.4,4$ and 6 for the aluminum-GFRP joint, $t_{i} / t_{e}=0.63,0.8,1.09,2$ and 3 for the aluminum-CFRP joint.

In particular, the metallic adherent has been realized by aluminum type 2024, whereas the composite adherent has been realized

Fig. 10. G-SIFs near point B, vs. $t_{i} / t_{e}$ for the double lap aluminum-GFRP co-cured joint. 


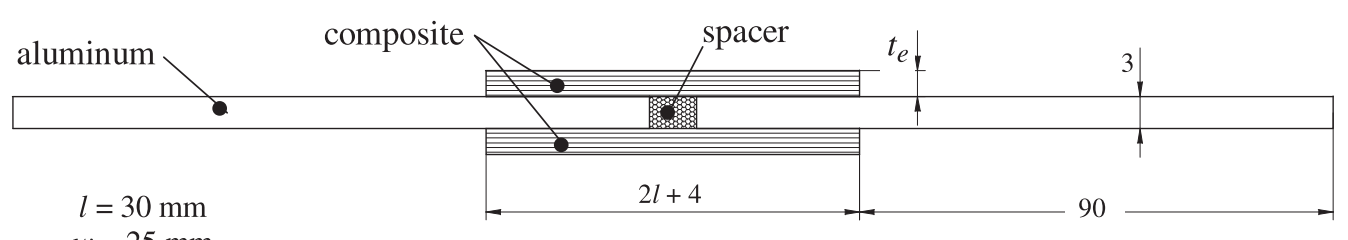

$w=25 \mathrm{~mm}$

Fig. 11. Geometry of double lap aluminum-composite co-cured joints used in the experimental tests.

Table 2

Elastic and geometrical properties of the adherents of the joints analyzed experimentally.

\begin{tabular}{lllll}
\hline Material & Thickness $(\mathrm{mm})$ & $\begin{array}{l}\text { Layers } \\
\text { number }\end{array}$ & $\begin{array}{l}E_{L}, E_{T}, G_{L T} \\
(\mathrm{GPa})\end{array}$ & $v_{L T}$ \\
\hline $\begin{array}{llll}\text { Aluminum } \\
\text { GFRP }\end{array}$ & 3.00 & 1 & 72.0 & 0.29 \\
& $0.50,0.75,1.25,2.25$, & $2,3,5,9,13$ & $32.9,6,2.1$ & 0.30 \\
CFRP & 3.25 & & & \\
& $1.00,1.50,2.75,3.75$, & $2,3,7,9,12$ & $155.5,6,2.1$ & 0.30 \\
& 4.75 & & & \\
\hline
\end{tabular}
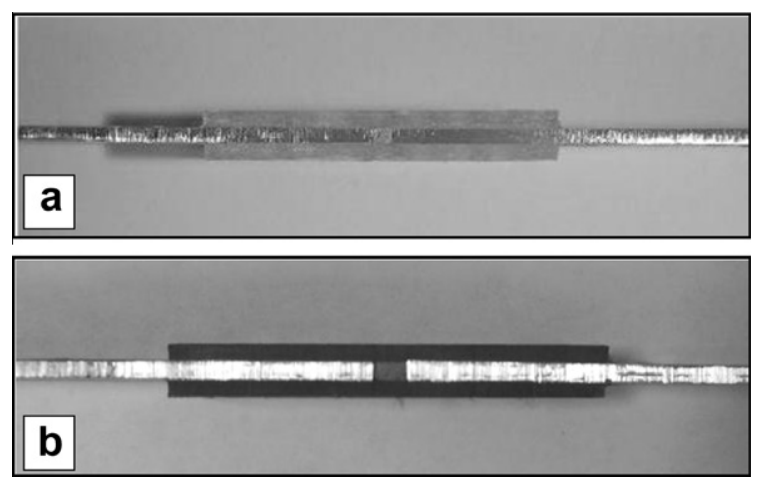

Fig. 12. Experimental analysis: image of double lap (a) aluminum-GFRP and (b) aluminum-CFRP joints.

by hand lay-up and unidirectional glass fiber type $\mathrm{E}\left(320 \mathrm{~g} / \mathrm{m}^{2}\right)$ for aluminum-GFRP joints, unidirectional carbon fiber type HM $\left(400 \mathrm{~g} / \mathrm{m}^{2}\right)$ for the aluminum-CFRP joints. In more detail, the GFRP unidirectional composite laminate is constituted by laminas having thickness of $0.25 \mathrm{~mm}$, the CFRP laminate is constituted instead by laminas having thickness of about $0.4-0.5 \mathrm{~mm}$.

Tables 1 and 2 show the elastic characteristics of the adherents, obtained by specific experimental tests carried out in accordance with the relative ASTM standards.

For each joint configuration, the tensile failure tests have been performed on four specimens (Fig. 12). From such tests it has been observed that for low $t_{i} / t_{e}$ values the damage mechanism involves always an adhesive failure that propagates from the attach point of the metallic adherent (point A, see Fig. 13a); on the contrary, for high $t_{i} / t_{e}$ values the adhesive failure propagates from the attach point of the composite adherent (point B, see Fig. 13b,c). Such a result is in a good agreement with the classical theory of bonded joint which predicts that the failure starts always from the attach point of the less stiff adherent [10].

Moreover, Fig. 14a shows the ultimate loads observed experimentally for the five aluminum-GFRP specimens having different $t_{i} / t_{e}$ ratio. It is possible to observe that the maximum ultimate load corresponds to $t_{i} / t_{e} \approx 2.5$. In particular, for low $t_{i} / t_{e}$ values the failure starts always from point $A$ and the ultimate load increases monotonically with $t_{i} / t_{e}$, whereas for a $t_{i} / t_{e}$ ratio higher than about 2.5 , failure starts always from the point $B$ and the ultimate load decreases slightly with $t_{i} / t_{e}$.

Also, by using the ultimate load of the aluminum-GFRP joints having $t_{i} / t_{e}=0.92$ (failure starting from point A with $\sigma_{u}=29.7 \mathrm{MPa}$ ) and $t_{i} / t_{e}=6$ (failure starting from the point B with $\sigma_{u}=71.8 \mathrm{MPa}$ ), for these joints Eq. (12a,b) provide $K_{1, A-c}=20.1 \mathrm{MPa} \mathrm{mm}^{0.285}$ and $K_{2, B-c}=12.3 \mathrm{MPa} \mathrm{mm}^{0.36}$, respectively.

In order to compare the experimental results with those obtained theoretically, Fig. 14b shows the curves of the ultimate load $L_{u, j}(j=A, B)$ evaluated by the proposed method, i.e. by Eqs. (13a,b), for failure that propagates from the point $\mathrm{A}\left(L_{u, A}\right)$ and from point $\mathrm{B}$ $\left(L_{u, B}\right)$. Obviously the area under the envelope of such last two curves corresponds to the strength domain described by Eq. (14). It is seen a very good accordance between the experimental results and the strength predictions provided by the proposed method. Also, the curves of the non-dimensional SIFs $\beta_{1, A}$ and $\beta_{2, B}$ (dominant SIF), represented in Fig. $14 \mathrm{~b}$, confirm that at the point $A$ the singular stress field increases with $t_{i} / t_{e}$ whereas the contrary occurs at the point B; consequently, for the two singular points the corresponding ultimate loads have opposite trend versus $t_{i} / t_{e}$. In particular, the $L_{u, A}$ curve intersect the $L_{u, B}$ curve at the $P_{L}$ point (absolute maximum static strength) whose abscissa $t_{i} / t_{e}$ is related to the particular coupled materials $\left(t_{i} / t_{e} \approx 2.5\right.$ for aluminum-GFRP).

Similarly, by using the ultimate load of the aluminum-CFRP joints having $t_{i} / t_{e}=0.63$ (failure at the point A with $\sigma_{u}=20.0 \mathrm{MPa}$ ) and $t_{i} / t_{e}=3$ (failure at the point B with $\sigma_{u}=33.33 \mathrm{MPa}$ ), for these
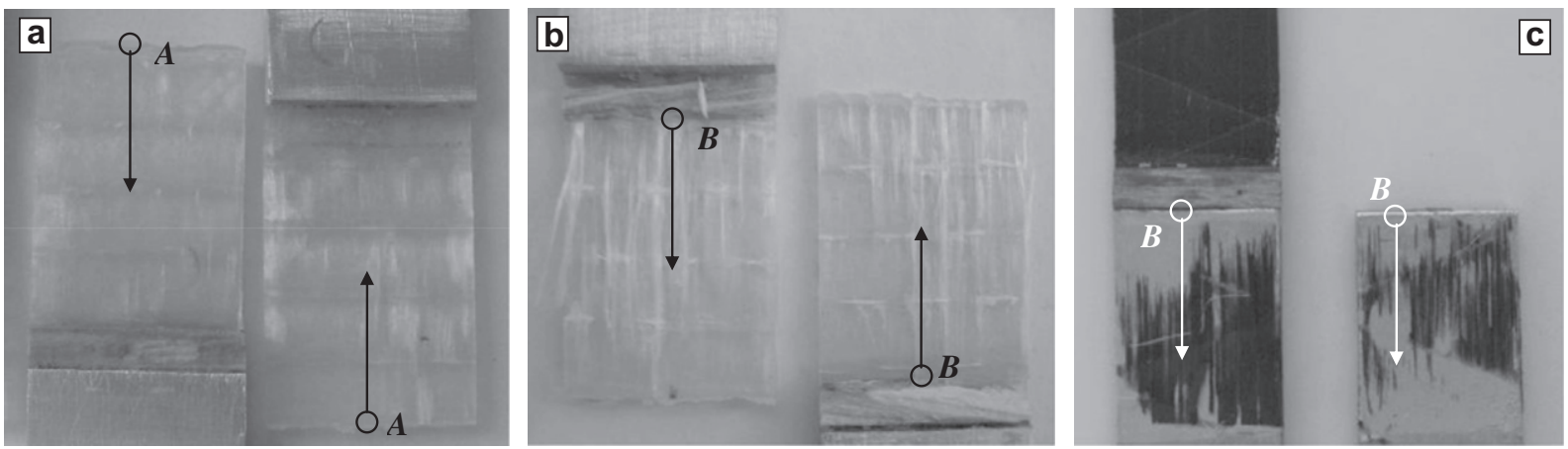

Fig. 13. Images of the typical failure surfaces for failure propagating (a) from point A and (b,c) from point B. 

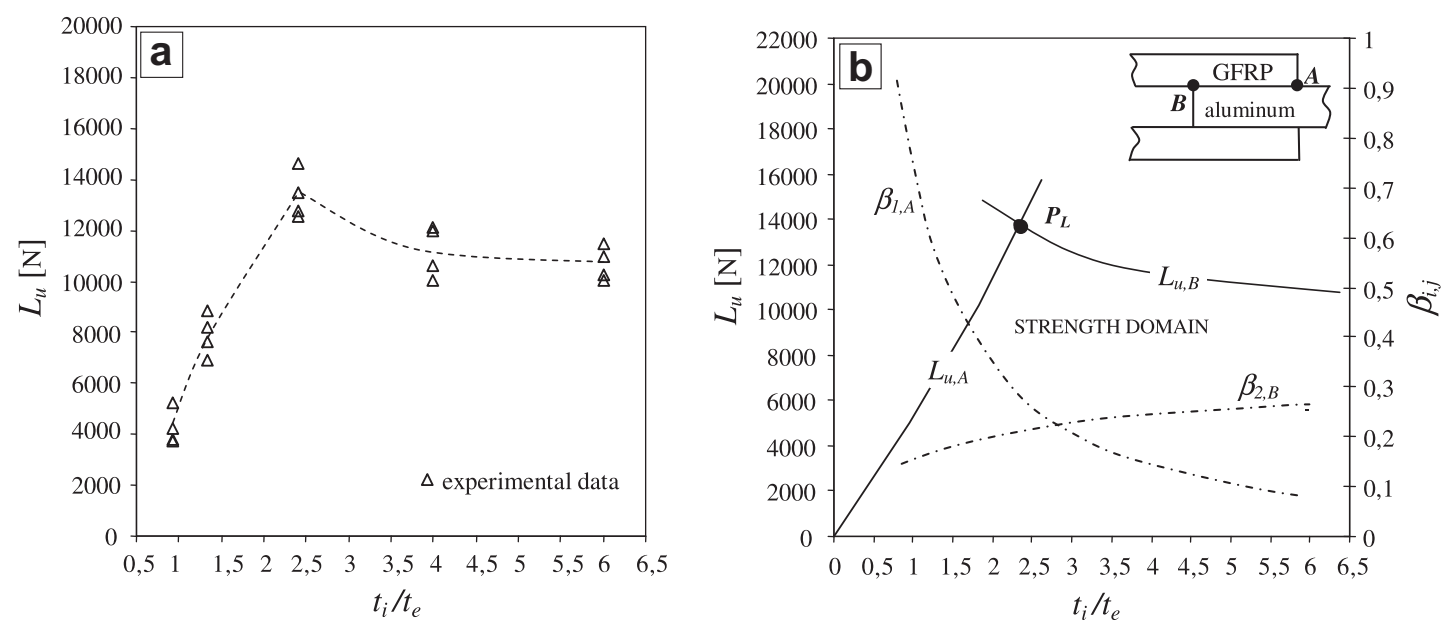

Fig. 14. Ultimate load vs. $t_{i} / t_{e}$ for aluminum-GFRP; (a) experimental results and (b) predictions provided by the proposed method.
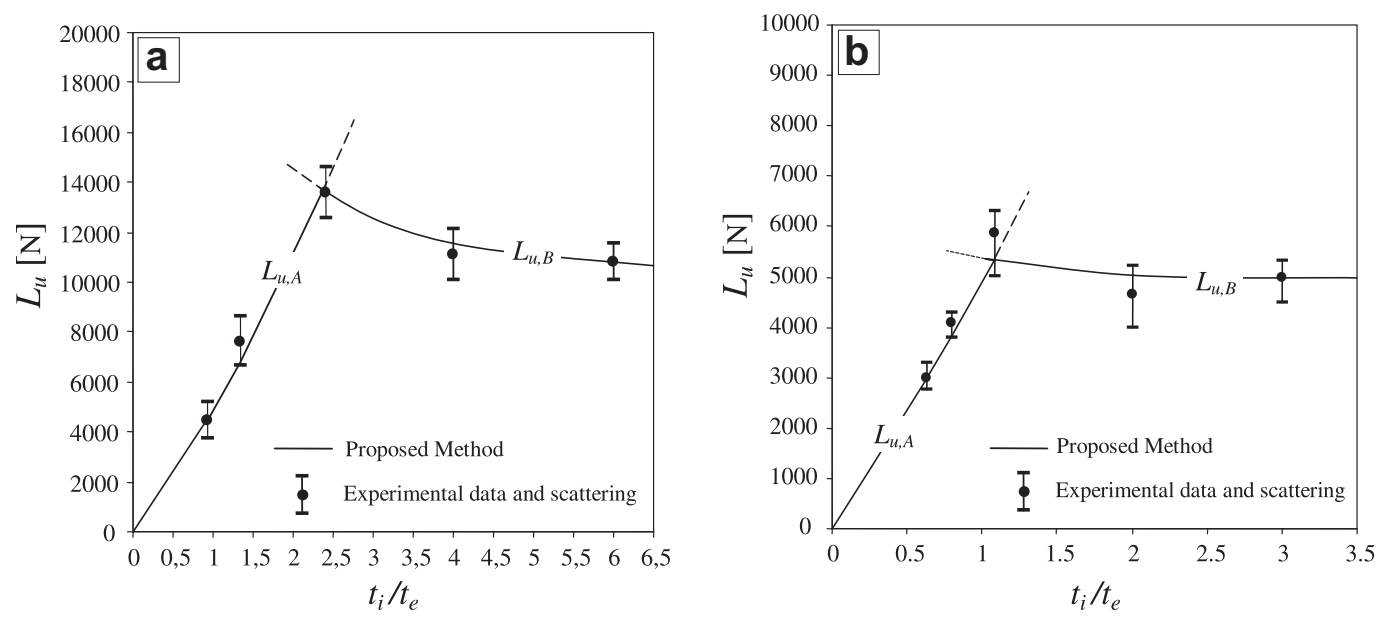

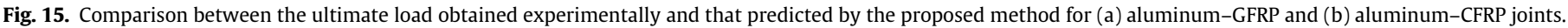

joints Eqs. (12a,b) provide $K_{1, A-c}=21.2 \mathrm{MPa} \mathrm{mm}^{0.321}$ and $K_{2, B-c}=$ $2.7 \mathrm{MPa} \mathrm{mm}^{0.279}$ respectively. By using these values Eqs. (13) provides the corresponding $L_{u, j}(j=A, B)$ curves.

Fig. 15 shows the good accordance between the experimental results and those provided by the proposed method for the aluminum-GFRP (Fig. 15a) and the aluminum-CFRP joints (Fig. 15b) examined.

\subsection{Comparison of the results relative to the different joints examined}

In order to compare the static strength of the joints Al-GFRP and Al-CFRP examined experimentally, in Fig. 16 the mean ultimate values versus $t_{i} / t_{e}$, are reported. It is seen that in practice for $t_{i} \mid t_{e}>1 \mathrm{Al}$-GFRP joint have a static strength always higher than that of the Al-CFRP joints.

Also, in accordance with the theoretical predictions it is observed that the abscissa $t_{i} / t_{e}$ of the intersect point $P_{L}$ increases with the mismatch of the elastic characteristics $\left(E_{i} / E_{e}\right.$ ratio) of the coupled adherents.

In particular, it is observed that by passing from an aluminumCFRP joint having $E_{i} / E_{e}=0.45$, to an aluminum-GFRP joint having $E_{i} / E_{e}=2.1$, the static strength is comparable for $t_{i} / t_{e} \leqslant 1$, whereas it redoubles for $t_{i} / t_{e} \geqslant 2$.

Also, it is possible to observe that for such hybrid metalliccomposite joints the increase of the thickness of the composite

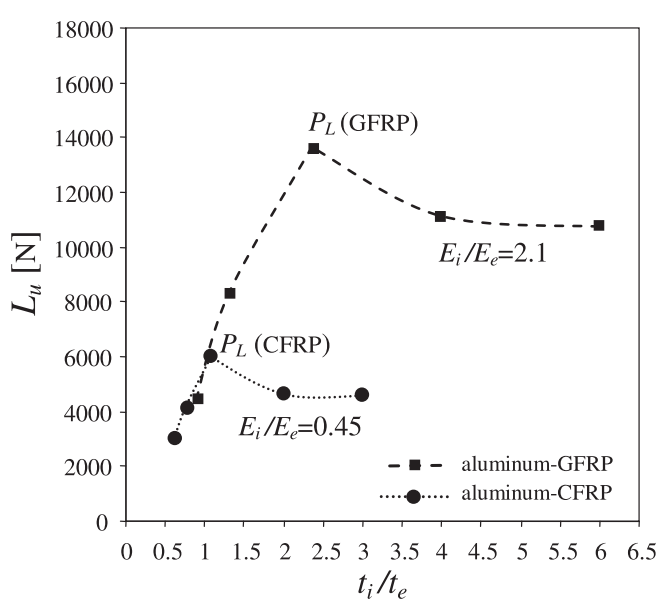

Fig. 16. Comparison between the ultimate loads obtained experimentally for aluminum-GFRP and aluminum-CFRP joints.

laminates in the overlap zone (as it is often performed in various practical applications, especially in the nautical field) leads to a little increase of the static strength of the joint only if the final value of $t_{i} / t_{e}$ is not less than the abscissa of the corresponding 


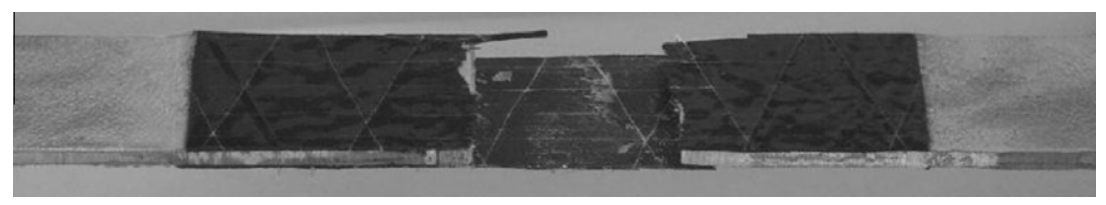

Fig. 17. Image of the typical failure of the aluminum-CFRP co-cured joint not influenced by the singular stress state.

Table 3

Results of the static failure tests for the aluminum-CFRP joint having $t_{e}=0.45 \mathrm{~mm}$.

\begin{tabular}{lllllll}
\hline$L_{u}(\mathrm{kN})$ & $b(\mathrm{~mm})$ & $l(\mathrm{~mm})$ & $t_{i}(\mathrm{~mm})$ & $t_{e}(\mathrm{~mm})$ & $\sigma_{u}(\mathrm{MPa})$ & $\sigma_{u, \text { CFRP }}(\mathrm{MPa})$ \\
\hline $17.7 \pm 5 \%$ & 25 & 30 & 3 & 0.45 & $236 \pm 5 \%$ & $787 \pm 5 \%$
\end{tabular}

point $P_{L}$ (2.5 and 1 respectively for Al-GFRP and Al-CFRP joints); otherwise such a practice leads to a significant decrease of the static strength and it has to be avoided; therefore in this last condition, for increasing the joint strength, it is better to increase the thickness $t_{i}$ of the internal metallic adherents in the overlap zone.

Finally, from the analysis of the experimental results it is possible to state that for a generic hybrid metal-composite joint the value of the geometrical characteristic ratio $t_{i} / t_{e}$ which corresponds to the maximum ultimate load moves from $t_{i} / t_{e} \approx 2.5$ to $t_{i} / t_{e} \approx 1$ by passing from GFRP to CFPR external adherents, i.e. from low modulus to high modulus composites. Intermediate values are obtained for joint with AFRP (kevlar, etc.) external adherents.

\subsection{Limitations of the proposed method}

Like in LEFM, the effectiveness of the proposed method, based on the G-SIFs, is related to a sufficient extension of the SDZ. In fact, if the extension of the SDZ is too small, then near the singular points, the unavoidable local plasticity phenomena make null the effects of the singularity which is not any more the parameter that governs the strength of the joints [37-43]. As an example, this condition happens for joints having external composite adherents with very small thickness, i.e. for joints with high $t_{i} / t_{e}$ values, as in the case of the examined double lap aluminum-CFRP joint having external composite adherents with $t_{e}=0.45 \mathrm{~mm}\left(t_{i} / t_{e}>6\right)$; for this joint it has been observed how the failure corresponds to the tensile failure of the CFRP adherents instead of the adhesive failure, although the ultimate load $L_{u, B}$ predicted by the proposed method (about $5000 \mathrm{~N}$ ) is smaller than tensile failure load of the CFRP adherents. Fig. 17 shows the typical failure of this joint that starts at the middle section of the composite adherent (where there are not shear stresses) and takes place for tensile stress very close the ultimate stress of the CFRP laminate that constitutes the external adherents. In detail, as it is reported in Table 3, the actual ultimate load of the joint is $L_{u}=17.7 \mathrm{kN}$ that is very close to the ultimate load of the CFRP adherents $L_{u, \text { CFRP }}=2 \cdot \sigma_{u, \text { CFRP }} \cdot b \cdot t_{e}=$ $2 \cdot 800 \mathrm{MPa} \cdot 25 \mathrm{~mm} \cdot 0.45 \mathrm{~mm}=18.0 \mathrm{kN}$, that is less than the ultimate load of the internal adherent $L_{u \text {,aluminum }}=\sigma_{u \text {,aluminum }} \cdot b t_{i}$ $=260 \mathrm{MPa} \cdot 25 \mathrm{~mm} \cdot 3 \mathrm{~mm}=19.5 \mathrm{kN}$. Such a result is in accordance with those reported in literature by various authors. As an example, qualitative study on the extension of the SDZ for bi-material joints have been performed in the last years by Akisanya and Fleck [42], as well as by Liu and Fleck [43] and also by Bogy [44] and Reedy Jr. [45]; by comparing the stress distributions obtained numerically and those obtained theoretically they estimate also the extension of the plastic zone for several load conditions.

For a better understanding of the above exposed phenomenon, numerical simulations of aluminum-CFRP joints have been carried

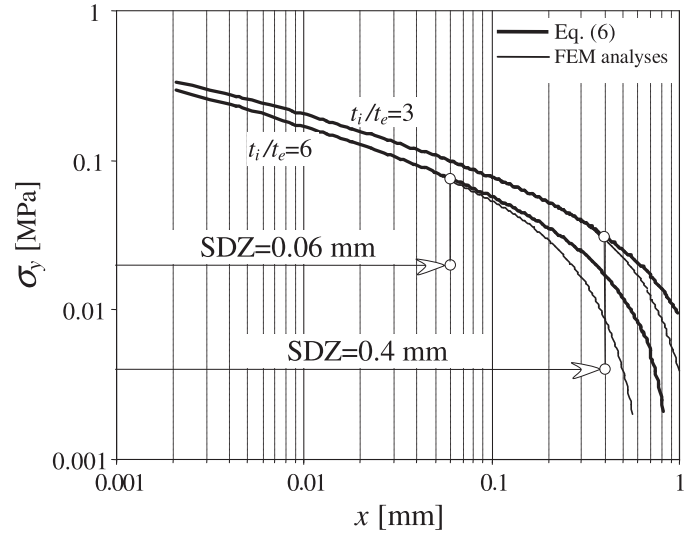

Fig. 18. Singular stress state near point $B$ of the aluminum-CFRP joint: extension of the SDZ for $t_{i} / t_{e}=6$ and $3 \mathrm{~mm}$.

out and the relative extension of the SDZ have been computed versus the CFRP adherent thickness. The results obtained from such analyses are depicted in a bi-logarithm scale in Fig. 18, along with the results obtained theoretically by Eq. (6), that refers to the point B (remember that for Al-CFRP joint having $t_{i} / t_{e}>1$ the proposed method predicts an adhesive failure starting from point $B$ ). It is seen how for $t_{i} / t_{e}=3$ the stress curve obtained by FEM coincides with the theoretical one until a distance of about $0.4 \mathrm{~mm}$ that therefore can be assumed as the typical extension of the SDZ; on the contrary, for $t_{i} / t_{e}=6$ the extension of the SDZ have a high reduction with values of about $0.06 \mathrm{~mm}$. Therefore, in accordance with the experimental results, it is possible to state that for hybrid double lap joints having $t_{i} / t_{e} \geqslant 6$ the extension of the SDZ becomes very small and the effects of the stress singularity are nullified from the unavoidable local plasticity phenomena. Such considerations and limitations confirm the validity of the proposed approach based on G-SIFs for the strength predictions of hybrid cocured joints.

\section{Conclusions}

In this work, hybrid double lap metal-composite co-cured joints have been studied theoretically, numerically and experimentally, by considering various materials for composite adherents (laminates reinforced with glass, aramid and carbon fibers) and metallic adherents (steel, aluminum and titanium). Such a materials selection permits in practice to cover all the range in which fall the joints used in the modern industrial production.

By means of the theoretical stress analysis performed by using the Lekhnitskii theory, the singularity orders of the singular stress field near the points where the joint interface intersects the free edge, have been computed by varying the main influence parameters. Successively, the G-SIFs have been evaluated by numerical simulations performed by means of the BEM.

From this systematic study it has been observed that the main characteristic parameters of the joint, as the taper angle of the free edge of the composite adherent, the elastic properties of the 
coupled materials, the geometrical ratio between the overlap length and the internal adherent thickness as well as the ratio between the thicknesses of the external adherent and the internal adherent, influence significantly the singular stress fields in the joint; also, their variations can lead to the transition of the most stressed zone from the attach point of the metallic adherent to the attach point of the composite adherent, with consequent significant variations of the static strength.

In detail, it has been observed that the static strength of a metal-composite co-cured joint increases not only with the taper angle of the composite adherent and the ratio between the overlap length and the internal metal adherent (up to $l / t_{i} \approx 6$ ) but also with the stiffness of the metallic adherent. On the contrary, it decreases with the longitudinal stiffness of the composite adherents. In detail, near the tapered edge of the external composite adherents the GSIF is related to the taper angle by a simple linear relationship, whereas the relationship with the ratio between the thickness of the internal adherent and that of the external adherents $\left(t_{i} / t_{e}\right)$ is represented by a simple exponential function. This last parameter in general influences significantly the static strength of the joint, since limited variations of it can lead to significant variations of the ultimate load. Also, when $t_{i} / t_{e}$ increases the point in which the typical adhesive failure starts, passes from the attach point of the composite adherent to the attach point of the metallic adherent.

The systematic analysis of such results have permitted to implement a new design method based on the G-SIFs, that allows the user the prediction of the static strength of the hybrid double lap co-cured joints.

Synthetically, by involving the singularity orders as well as the dominant non-dimensional SIFs of each singular point, the proposed method allows the user to compare the actual applied load with the critical SIFs determined experimentally by using a simple specimen; in such a way it permits to take into account all the manufacture parameters (as roughness and surface treatments, curing conditions, etc.) that, as it is well known, influence significantly the mechanical behavior of a bonded joint. Also, for any joint configuration it allows the user to determine the optimal adherent thickness ratio which corresponds to the absolute maximum load; consequently, it gives to the designer the correct indications about the most convenient modifications of the thickness of the adherents near the overlap zone, to optimize the static strength. In order to assess both theoretical and numerical results in terms of G-SIFs, as well as to test the accuracy of the proposed method, systematic static failure tests on aluminum-GFRP and aluminum-CFRP cocured joints, have been carried out by varying the ratio between the thickness of the coupled adherents.

The results obtained have confirmed the accuracy of the theoretical and numerical analyses performed in this work as well as the reliability and the limitations of the proposed design method based on the G-SIFs. Like LEFM the main limitation of the proposed method is related to the extension of the SDZ: if it is too small, then unavoidable local plasticity nullifies the effects of the stress singularity and, consequently, the joint failure is related to other damage mechanisms. For the examined hybrid joints such a condition occurs only when the external composite adherents thickness $t_{e}$ is very small, i.e. when $t_{i} / t_{e}$ is very high (in general $t_{i} / t_{e}>6$ ), and the joint failure follows the tensile failure of the composite adherents.

\section{Appendix A}

The theoretical analysis exposed in chapter 2 is based on the complex potentials, i.e. on the three complex functions $\Phi_{1, i}\left(z_{1 i}\right)$, $\Phi_{2, i}\left(z_{2 i}\right)$ and $\Phi_{3, i}\left(z_{3 i}\right)$, defined as:
$\Phi_{1, \mathrm{i}}\left(z_{1 \mathrm{i}}\right)=a_{1 i} z_{1 i}^{\lambda}+a_{2 i} z_{1 i}^{\bar{\lambda}}$

$\Phi_{2, \mathrm{i}}\left(z_{2 \mathrm{i}}\right)=b_{1 i} z_{2 i}^{\lambda}+b_{2 i} z_{2 i}^{\bar{\lambda}}$

$\Phi_{3, i}\left(z_{3 i}\right)=c_{1 i} z_{3 i}^{\lambda}+c_{2 i} z_{3 i}^{\bar{\lambda}} \quad i=1,2$

In (A.1) the subscript $i$ refers to the material considered, $a_{j i}, b_{j i}$ and $c_{j i}$ $(i=1,2$ and $j=1,2,3)$ are the unknown coefficients, whereas $z_{k i}$ $(k=1,2,3)$ are the complex variables related to the $x$ and $y$ coordinates by means of the following relationship:

$z_{k i}=x+\mu_{k i} y \quad(k=1,2,3)$

In Eq. (A.2), the terms $\mu_{k i}(k=1,2,3)$ are the solutions of the characteristic equation associated to the differential equation that describes the examined elastic problem [16-19]:

$l_{4}\left(\mu_{k i}\right) \cdot l_{2}\left(\mu_{k i}\right)-l_{3}^{2}\left(\mu_{k i}\right)=0$

In Eq. (A.3) the functions $l_{2}\left(\mu_{k i}\right), l_{3}\left(\mu_{k i}\right)$ and $l_{4}\left(\mu_{k i}\right)$ are expressed by the following relationships:

$$
\begin{aligned}
& l_{2}\left(\mu_{k i}\right)=\beta_{55} \mu_{k i}^{2}-2 \beta_{45} \mu_{k i}+\beta_{44} \\
& l_{3}\left(\mu_{k i}\right)=\beta_{15} \mu_{k i}^{3}-\left(\beta_{14}+\beta_{56}\right) \mu_{k i}^{2}+\left(\beta_{25}+\beta_{46}\right) \mu_{k i}-\beta_{24} \\
& l_{4}\left(\mu_{k i}\right)=\beta_{11} \mu_{k i}^{4}-2 \beta_{16} \mu_{k i}^{3}+\left(2 \beta_{12}+\beta_{66}\right) \mu_{k i}^{2}-2 \beta_{26} \mu_{k i}+\beta_{22}
\end{aligned}
$$

The $\beta_{l m}(l=1-6$ and $m=1-6)$ constants that appear into Eqs. (A.4) are related to the terms $A_{l m}(l=1-6$ and $m=1-6)$ of the compliance matrix $A$ of the materials involved in the stress-strain relationship:

$$
\left[\begin{array}{c}
\varepsilon_{x} \\
\varepsilon_{y} \\
\varepsilon_{z} \\
\gamma_{y z} \\
\gamma_{x z} \\
\gamma_{x y}
\end{array}\right]_{i}=\left[\begin{array}{llllll}
A_{11} & A_{12} & A_{13} & A_{14} & A_{15} & A_{16} \\
A_{21} & A_{22} & A_{23} & A_{24} & A_{25} & A_{26} \\
A_{31} & A_{32} & A_{33} & A_{34} & A_{35} & A_{36} \\
A_{41} & A_{42} & A_{43} & A_{44} & A_{45} & A_{46} \\
A_{51} & A_{52} & A_{53} & A_{54} & A_{55} & A_{56} \\
A_{61} & A_{62} & A_{63} & A_{64} & A_{65} & A_{66}
\end{array}\right]_{i}\left[\begin{array}{c}
\sigma_{x} \\
\sigma_{y} \\
\sigma_{z} \\
\tau_{y z} \\
\tau_{x z} \\
\tau_{x y}
\end{array}\right]_{i} \quad i=1,2
$$

In detail, the constants are related to the matrix elements by the relationship:

$\left(\beta_{l m}\right)_{i}=\left(A_{l m}-\frac{A_{l 3} \cdot A_{m 3}}{A_{33}}\right)_{i} \quad(l, m=1,2, \ldots, 6) \quad(i=1,2)$

Eq. (A.3) has in general six complex solutions that can be written by using the following simple notations:

$$
\left\{\begin{array}{lll}
\mu_{1 i}=\alpha_{1}+i \beta_{1} ; & \mu_{2 i}=\alpha_{2}+i \beta_{2} ; & \mu_{3 i}=\alpha_{3}+i \beta_{3} \\
\bar{\mu}_{1 i}=\alpha_{1}-i \beta_{1} ; & \bar{\mu}_{2 i}=\alpha_{2}-i \beta_{2} ; & \bar{\mu}_{3 i}=\alpha_{3}-i \beta_{3}
\end{array}\right.
$$

Once the parameters $\mu_{k i}$ and the functions $\Phi_{j i}(j=1,2$ and $i=1,2,3)$ are evaluated, then the stress components $\sigma_{x, i}, \sigma_{y, i}$ and $\tau_{x y, i}$ are described by the relationships:

$$
\left\{\begin{array}{l}
\sigma_{x, i}=2 \cdot \operatorname{Re}\left[\mu_{1, i}^{2} \cdot \Phi_{1, i}^{\prime}\left(z_{1, i}\right)+\mu_{2, i}^{2} \cdot \Phi_{2, i}^{\prime}\left(z_{2, i}\right)+\mu_{3, i}^{2} \cdot \xi_{3, i} \cdot \Phi_{3, i}^{\prime}\left(z_{3, i}\right)\right] \\
\sigma_{y, i}=2 \cdot \operatorname{Re}\left[\Phi_{1, i}^{\prime}\left(z_{1, i}\right)+\Phi_{2, i}^{\prime}\left(z_{2, i}\right)+\xi_{3, i} \cdot \Phi_{3, i}^{\prime}\left(z_{3, i}\right)\right] \\
\tau_{x y, i}=-2 \cdot \operatorname{Re}\left[\mu_{1, i} \cdot \Phi_{1, i}^{\prime}\left(z_{1, i}\right)+\mu_{2, i} \cdot \Phi_{2, i}^{\prime}\left(z_{2, i}\right)+\mu_{3, i} \cdot \xi_{3, i} \cdot \Phi_{3, i}^{\prime}\left(z_{3, i}\right)\right]
\end{array} \quad i=1,2\right.
$$

The displacement components $u_{i}, v_{i}$ and $w_{i}$ are instead given from:

$$
\left\{\begin{array}{l}
u_{i}=2 \cdot \operatorname{Re}\left[\sum_{k=1}^{3} p_{k, i} \cdot \Phi_{k, i}\left(z_{k, i}\right)\right] \\
v_{i}=2 \cdot \operatorname{Re}\left[\sum_{k=1}^{3} q_{k, i} \cdot \Phi_{k, i}\left(z_{k, i}\right)\right] \quad(i=1,2) \\
w_{i}=2 \cdot \operatorname{Re}\left[\sum_{k=1}^{3} r_{k, i} \cdot \Phi_{k, i}\left(z_{k, i}\right)\right]
\end{array}\right.
$$

Finally, the functions $p_{k, i}, q_{k, i}$ and $r_{k, i}(k=1,2)$ that appears into Eqs. (A.9) are related to the constants $\left(\beta_{l m}\right)_{i}$ and $\mu_{k, i}$ by the relationships: 
$\left\{\begin{array}{l}p_{k, i}=\beta_{11, i} \mu_{k, i}^{2}+\beta_{12, i}-\beta_{16, i} \mu_{k, i}+\xi_{k, i}\left(\beta_{15, i} \mu_{k, i}-\beta_{14, i}\right) \\ q_{k, i}=\beta_{12, i} \mu_{k, i}+\frac{\beta_{22, i}}{\mu_{k, i}}-\beta_{26, i}+\xi_{k, i}\left(\beta_{25, i}-\frac{\beta_{24, i}}{\mu_{k, i}}\right) \\ r_{k, i}=\beta_{14, i} \mu_{k, i}+\frac{\beta_{24, i}}{\mu_{k, i}}-\beta_{46, i}+\xi_{k, i}\left(\beta_{45, i}-\frac{\beta_{44, i}}{\mu_{k, i}}\right)\end{array} \quad(i=1,2)\right.$

(A.10a)

whereas for $k=3$, it follows:

$$
\left\{\begin{array}{l}
p_{3, i}=\xi_{3, i} \cdot\left(\beta_{11, i} \mu_{3, i}^{2}+\beta_{12, i}-\beta_{16, i} \mu_{3, i}\right)+\beta_{15, i} \mu_{3, i}-\beta_{14, i} \\
q_{3, i}=\xi_{3, i} \cdot\left(\beta_{12, i} \mu_{3, i}+\frac{\beta_{22, i}}{\mu_{3, i}}-\beta_{26, i}\right)+\beta_{25, i}-\frac{\beta_{24, i}}{\mu_{3, i}} \\
r_{3, i}=\xi_{3, i}\left(\beta_{14, i} \mu_{3, i}+\frac{\beta_{24, i}}{\mu_{3, i}}-\beta_{46, i}\right)+\beta_{45, i}-\frac{\beta_{44, i}}{\mu_{3, i}}
\end{array} \quad(i=1,2)\right.
$$

In Eqs. (A.10) the following relationships can be applied:

$\xi_{k, i}=-\frac{l_{3, i}\left(\mu_{k, i}\right)}{l_{2, i}\left(\mu_{k, i}\right)} ; \quad \xi_{3, i}=-\frac{l_{3, i}\left(\mu_{3, i}\right)}{l_{4, i}\left(\mu_{3, i}\right)} \quad(k=1,2) \quad(i=1,2)$

(A.11a, b)

To solve the elastic problem related to the co-cured joints, a cylindrical reference system $(r, \theta, s)$ with reference axis lying on the interface between the two coupled materials can be considered (as an example see Fig. 1b for point $A)$.

By substituting Eqs. (A.8) and (A.9) in the well known rotation relationships for stresses and displacements, and by assuming a plain strain state, it follows that:

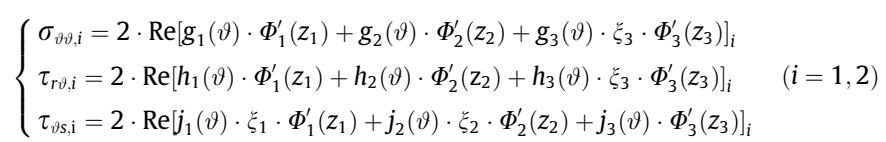

$$
\left\{\begin{array}{l}
u_{r, i}=2 \cdot \operatorname{Re}\left[\sum_{\mathrm{k}=1}^{3}\left(e_{\mathrm{k}}(\vartheta) \cdot \Phi_{\mathrm{k}}\left(z_{\mathrm{k}}\right)\right)\right]_{i} \\
u_{\vartheta, i}=2 \cdot \operatorname{Re}\left[\sum_{\mathrm{k}=1}^{3}\left(f_{\mathrm{k}}(\vartheta) \cdot \Phi_{\mathrm{k}}\left(z_{\mathrm{k}}\right)\right)\right]_{i}(i=1,2) \\
u_{s, i}=2 \cdot \operatorname{Re}\left[\sum_{\mathrm{k}=1}^{3}\left(r_{\mathrm{k}} \Phi_{\mathrm{k}}\left(z_{\mathrm{k}}\right)\right)\right]_{i}
\end{array}\right.
$$

being:

$$
\left\{\begin{array}{l}
z_{k, i}=r\left(\cos \vartheta+\mu_{k, i} \sin \vartheta\right) \equiv r \zeta_{i j}(\vartheta) \\
g_{k, i}(\vartheta)=\sin ^{2} \vartheta \cdot \mu_{k, i}^{2}+\cos ^{2} \vartheta+2 \cdot \sin \vartheta \cdot \cos \vartheta \cdot \mu_{k, i} \\
h_{k, i}(\vartheta)=-\sin \vartheta \cdot \cos \vartheta \cdot \mu_{k, i}^{2}+\sin \vartheta \cdot \cos \vartheta+\left(\sin ^{2} \vartheta+\cos ^{2} \vartheta\right) \cdot \mu_{k, i}^{2} \quad(i=1,2) \\
j_{k, i}(\vartheta)=\sin \vartheta \cdot \mu_{k, i}^{+} \cos \vartheta \\
e_{k, i}(\vartheta)=\cos \vartheta \cdot p_{k, i}+\sin \vartheta \cdot q_{k, i} \\
f_{k, i}(\vartheta)=\sin \vartheta \cdot p_{k, i}-\cos \vartheta \cdot q_{k, i}
\end{array}\right.
$$

where the angular functions $f_{i j}^{v}(\theta)$ and $h_{i}^{v}(\theta)$ of Eq. (1) are expressed by the relationships (see Appendix B):

$$
\left\{\begin{array}{l}
f_{k j, i}^{v}(\vartheta)=f_{i j, i}\left(a_{1, i}, \bar{a}_{2, i}, b_{1, i}, \bar{b}_{2, i}, c_{1, i}, \bar{c}_{2, i}, \omega, \vartheta\right) \\
h_{k, i}^{v}(\vartheta)=h_{i, i}\left(a_{1, i}, \bar{a}_{2, i}, b_{1, i}, \bar{b}_{2, i}, c_{1, i}, \bar{c}_{2, i}, \omega, \vartheta\right)
\end{array} \quad(k, j=r, \theta, s) \quad(i=1,2)\right.
$$

Into Eqs. (A.15) the terms $a_{1, i}, a_{2, i}, b_{1, i}, b_{2, i}, c_{1, i}, c_{2, i}$ are the unknowns relative to the two coupled materials. By imposing the boundary conditions (outlined in Section 2) around point A of Fig. 1a, the following set of 12 homogeneous equations is obtained:

$$
\left\{\begin{array}{l}
\sigma_{\vartheta \vartheta, 1}(r, 0)=0 \\
\tau_{\mathrm{r} \vartheta, 1}(r, 0)=0 \\
\tau_{\vartheta \mathrm{s}, 1}(r, 0)=0 \\
\sigma_{\vartheta \vartheta, 2}(r, \varphi)=0 \\
\tau_{\mathrm{r} \vartheta, 2}(r, \varphi)=0 \\
\tau_{\vartheta \mathrm{s}, 2}(r, \varphi)=0 \\
\sigma_{\vartheta \vartheta, 1}(r, \pi)-\sigma_{\vartheta \vartheta, 2}(r, \pi)=0 \\
\tau_{\mathrm{r} \vartheta, 1}(r, \pi)-\tau_{\mathrm{r} \vartheta, 2}(r, \pi)=0 \\
\tau_{\vartheta \mathrm{s}, 1}(r, \pi)-\tau_{\vartheta \mathrm{s}, 2}(r, \pi)=0 \\
u_{\mathrm{r}, 1}(r, \pi)-u_{\mathrm{r}, 2}(r, \pi)=0 \\
u_{\vartheta, 1}(r, \pi)-u_{\vartheta, 2}(r, \pi)=0 \\
u_{\mathrm{s}, 1}(r, \pi)-u_{\mathrm{s}, 2}(r, \pi)=0
\end{array}\right.
$$

Analogous set of boundary conditions can be written around point $B$ (see Fig. 1a); it is obtained by substituting the subscript 1 with the subscript 2 and by imposing $\varphi=90^{\circ}$.

Finally, by substituting Eqs. (A.15) into Eqs. (1) and this last into Eqs. (A.16), an homogeneous equation set involving the 12 unknowns $a_{1, i}, a_{2, i}, b_{1, i}, b_{2, i}, c_{1, i}, c_{2, i}(i=1,2)$ as well as the unknown singularity order is obtained (see Eq. (2)).

\section{Appendix B}

The elements $m_{i j}(i, j=1,2, \ldots, 12)$ of the matrix $[M(\omega)]$ are here reported:

$$
\begin{aligned}
\left\{m_{1 j}\right\}= & \left\{g_{11}(0) \zeta_{11}^{\omega-1}(0), \bar{g}_{11}(0) \bar{\zeta}_{11}^{\omega-1}(0), g_{21}(0) \zeta_{21}^{\omega-1}(0), \bar{g}_{21}(0) \bar{\zeta}_{21}^{\omega-1}(0)\right. \\
& \left.g_{31}(0) \xi_{31} \zeta_{31}^{\omega-1}(0), \bar{g}_{31}(0) \bar{\xi}_{31} \bar{\zeta}_{31}^{\omega-1}(0), 0,0,0,0,0,0\right\}
\end{aligned}
$$

$\left\{m_{2 j}\right\}=\left\{h_{11}(0) \zeta_{11}^{\omega-1}(0), \bar{h}_{11}(0) \bar{\zeta}_{11}^{\omega-1}(0), h_{21}(0) \zeta_{21}^{\omega-1}(0), \bar{h}_{21}(0) \bar{\zeta}_{21}^{\omega-1}(0)\right.$,

$$
\left.h_{31}(0) \xi_{31} \zeta_{31}^{\omega-1}(0), \bar{h}_{31}(0) \bar{\xi}_{31} \bar{\zeta}_{31}^{\omega-1}(0), 0,0,0,0,0,0\right\}
$$

$$
\left\{m_{3 j}\right\}=\left\{j_{11}(0) \zeta_{11}^{\omega-1}(0), \bar{j}_{11}(0) \bar{\zeta}_{11}^{\omega-1}(0), j_{21}(0) \zeta_{21}^{\omega-1}(0), \bar{j}_{21}(0) \bar{\zeta}_{21}^{\omega-1}(0)\right.
$$$$
\left.j_{31}(0) \xi_{31} \zeta_{31}^{(\omega-1}(0), \bar{j}_{31}(0) \bar{\xi}_{31} \bar{\zeta}_{31}^{(\omega-1}(0), 0,0,0,0,0,0\right\}
$$

$\left\{m_{4 j}\right\}=\left\{0,0,0,0,0,0, g_{12}(\vartheta) \zeta_{12}^{\omega-1}(\vartheta), \bar{g}_{12}(\vartheta) \bar{\zeta}_{12}^{\omega-1}(\vartheta), g_{22}(\vartheta) \zeta_{22}^{\omega-1}(\vartheta)\right.$,

$$
\left.\bar{g}_{22}(\vartheta) \bar{\zeta}_{22}^{\omega-1}(\vartheta), g_{32}(\vartheta) \xi_{32} \zeta_{32}^{\omega-1}(\vartheta), \bar{g}_{32}(\vartheta) \bar{\xi}_{32} \bar{\zeta}_{32}^{\omega-1}(\vartheta)\right\}
$$

$\left\{m_{5 j}\right\}=\left\{0,0,0,0,0,0, h_{12}(\vartheta) \zeta_{12}^{\omega-1}(\vartheta), \bar{h}_{12}(\vartheta) \bar{\zeta}_{12}^{\omega-1}(\vartheta), h_{22}(\vartheta) \zeta_{22}^{\omega-1}(\vartheta)\right.$,

$$
\left.\bar{h}_{22}(\vartheta) \bar{\zeta}_{22}^{(\omega-1}(\vartheta), h_{32}(\vartheta) \xi_{32} \zeta_{32}^{(\omega-1}(\vartheta), \bar{h}_{32}(\vartheta) \bar{\xi}_{32} \bar{\zeta}_{32}^{(-1}(\vartheta)\right\}
$$

$$
\left\{m_{6 j}\right\}=\left\{0,0,0,0,0,0, j_{12}(\vartheta) \zeta_{12}^{\omega-1}(\vartheta), \bar{j}_{12}(\vartheta) \bar{\zeta}_{12}^{\omega-1}(\vartheta), j_{22}(\vartheta) \zeta_{22}^{\omega-1}(\vartheta),\right.
$$$$
\left.\bar{j}_{22}(\vartheta) \bar{\zeta}_{22}^{(\omega-1}(\vartheta), j_{32}(\vartheta) \xi_{32} \zeta_{32}^{\omega-1}(\vartheta), \bar{j}_{32}(\vartheta) \bar{\xi}_{32} \bar{\zeta}_{32}^{\omega-1}(\vartheta)\right\}
$$

$$
\begin{aligned}
\left\{m_{7 j}\right\}= & \left\{g_{11}(\pi) \zeta_{11}^{\omega-1}(\pi), \bar{g}_{11}(\pi) \bar{\zeta}_{11}^{\omega-1}(\pi), g_{21}(\pi) \zeta_{21}^{\omega-1}(\pi), \bar{g}_{21}(\pi) \bar{\zeta}_{21}^{\omega-1}(\pi),\right. \\
& g_{31}(\pi) \xi_{31} \zeta_{31}^{\omega-1}(\pi), \bar{g}_{31}(\pi) \bar{\xi}_{31} \bar{\zeta}_{31}^{\omega-1}(\pi),-g_{12}(\pi) \zeta_{12}^{\omega-1}(\pi), \\
& -\bar{g}_{12}(\pi) \bar{\zeta}_{12}^{\omega-1}(\pi),-g_{22}(\pi) \zeta_{22}^{\omega-1}(\pi),-\bar{g}_{22}(\pi) \bar{\zeta}_{22}^{\omega-1}(\pi), \\
& \left.-g_{32}(\pi) \xi_{32} \zeta_{32}^{\omega-1}(\pi),-\bar{g}_{32}(\pi) \bar{\xi}_{32} \bar{\zeta}_{32}^{\omega-1}(\pi)\right\} \\
\left\{m_{8 j}\right\}= & \left\{h_{11}(\pi) \zeta_{11}^{\omega-1}(\pi), \bar{h}_{11}(\pi) \bar{\zeta}_{11}^{\omega-1}(\pi), h_{21}(\pi) \zeta_{21}^{\omega-1}(\pi), \bar{h}_{21}(\pi) \bar{\zeta}_{21}^{\omega-1}(\pi),\right. \\
& h_{31}(\pi) \xi_{31} \zeta_{31}^{\omega-1}(\pi), \bar{h}_{31}(\pi) \bar{\xi}_{31} \bar{\zeta}_{31}^{\omega-1}(\pi),-h_{12}(\pi) \zeta_{12}^{(\omega-1}(\pi), \\
& -\bar{h}_{12}(\pi) \bar{\zeta}_{12}^{\omega-1}(\pi),-h_{22}(\pi) \zeta_{22}^{\omega-1}(\pi),-\bar{h}_{22}(\pi) \bar{\zeta}_{22}^{\omega-1}(\pi), \\
& \left.-h_{32}(\pi) \xi_{32} \zeta_{32}^{\omega-1}(\pi),-\bar{h}_{32}(\pi) \bar{\xi}_{32} \bar{\zeta}_{32}^{\omega-1}(\pi)\right\} \\
\left\{m_{9 j}\right\}= & \left\{j_{11}(\pi) \zeta_{11}^{\omega-1}(\pi), \bar{j}_{11}(\pi) \bar{\zeta}_{11}^{\omega-1}(\pi), j_{21}(\pi) \zeta_{21}^{\omega-1}(\pi), \bar{j}_{21}(\pi) \bar{\zeta}_{21}^{\omega-1}(\pi),\right. \\
& j_{31}(\pi) \xi_{31} \zeta_{31}^{\omega-1}(\pi), \bar{j}_{31}(\pi) \bar{\xi}_{31} \bar{\zeta}_{31}^{\omega-1}(\pi),-j_{12}(\pi) \zeta_{12}^{\omega-1}(\pi), \\
& -\bar{j}_{12}(\pi) \bar{\zeta}_{12}^{\omega-1}(\pi),-j_{22}(\pi) \zeta_{22}^{\omega-1}(\pi),-\bar{j}_{22}(\pi) \bar{\zeta}_{22}^{\omega-1}(\pi), \\
& \left.-j_{32}(\pi) \xi_{32} \zeta_{32}^{\omega-1}(\pi),-\bar{j}_{32}(\pi) \bar{\xi}_{32} \bar{\zeta}_{32}^{\omega-1}(\pi)\right\}
\end{aligned}
$$




$$
\begin{aligned}
\left\{m_{10 j}\right\}= & \left\{e_{11}(\pi) \zeta_{11}^{\omega}(\pi), \bar{e}_{11}(\pi) \bar{\zeta}_{11}^{\omega}(\pi), e_{21}(\pi) \zeta_{21}^{\omega}(\pi), \bar{e}_{21}(\pi) \bar{\zeta}_{21}^{\omega}(\pi),\right. \\
& e_{31}(\pi) \zeta_{31}^{\omega}(\pi), \bar{e}_{31}(\pi) \bar{\zeta}_{31}^{\omega}(\pi),-e_{12}(\pi) \zeta_{12}^{\omega}(\pi),-\bar{e}_{12}(\pi) \bar{\zeta}_{12}^{\omega}(\pi), \\
& -e_{22}(\pi) \zeta_{22}^{\omega}(\pi),-\bar{e}_{22}(\pi) \bar{\zeta}_{22}^{\omega}(\pi),-e_{32}(\pi) \zeta_{32}^{\omega}(\pi), \\
& \left.-\bar{e}_{32}(\pi) \bar{\zeta}_{32}^{\omega}(\pi)\right\} \\
\left\{m_{11 j}\right\}= & \left\{f_{11}(\pi) \zeta_{11}^{\omega}(\pi), \bar{f}_{11}(\pi) \bar{\zeta}_{11}^{\omega}(\pi), f_{21}(\pi) \zeta_{21}^{\omega}(\pi), \bar{f}_{21}(\pi) \bar{\zeta}_{21}^{\omega}(\pi),\right. \\
& f_{31}(\pi) \zeta_{31}^{\omega}(\pi), \bar{f}_{31}(\pi) \bar{\zeta}_{31}^{\omega}(\pi),-f_{12}(\pi) \zeta_{12}^{\omega}(\pi),-\bar{f}_{12}(\pi) \bar{\zeta}_{12}^{\omega}(\pi), \\
& -f_{22}(\pi) \zeta_{22}^{\omega}(\pi),-\bar{f}_{22}(\pi) \bar{\zeta}_{22}^{\omega}(\pi),-f_{32}(\pi) \zeta_{32}^{\omega}(\pi), \\
& \left.-\bar{f}_{32}(\pi) \bar{\zeta}_{32}^{\omega}(\pi)\right\} \\
\left\{m_{12 j}\right\}= & \left\{r_{11}(\pi) \zeta_{11}^{\omega}(\pi), \bar{r}_{11}(\pi) \bar{\zeta}_{11}^{\omega}(\pi), r_{21}(\pi) \zeta_{21}^{\omega}(\pi), \bar{r}_{21}(\pi) \bar{\zeta}_{21}^{\omega}(\pi),\right. \\
& r_{31}(\pi) \zeta_{31}^{\omega}(\pi), \bar{r}_{31}(\pi) \bar{\zeta}_{31}^{\omega}(\pi),-r_{12}(\pi) \zeta_{12}^{\omega}(\pi),-\bar{r}_{12}(\pi) \bar{\zeta}_{12}^{\omega}(\pi), \\
& -r_{22}(\pi) \zeta_{22}^{\omega}(\pi),-\bar{r}_{22}(\pi) \bar{\zeta}_{22}^{\omega}(\pi),-r_{32}(\pi) \zeta_{32}^{\omega}(\pi), \\
& \left.-\bar{r}_{32}(\pi) \bar{\zeta}_{32}^{\omega}(\pi)\right\}
\end{aligned}
$$

\section{References}

[1] Ascione F, Feo L, Maceri F. An experimental investigation on the bearing failure load of glass fibre/epoxy laminates. Compos Part B: Eng 2009;40(3):197-205.

[2] Ascione F, Feo L, Maceri F. On the pin-bearing failure load of GFRP bolted laminates: an experimental analysis on the influence of bolt diameter. Compos Part B: Eng 2010;41(6):482-90.

[3] Shin KC, Lee JJ. Prediction of the tensile load-bearing capacity of a co-cured single lap joint considering residual thermal stresses. J Adhes Sci Technol 2000;14(13):1691-704.

[4] Shin KC, Lee JJ. Tensile load-bearing capacity of co-cured double lap joints. J Adhes Sci Technol 2000;14(12):1539-56.

[5] Shin KC, Lee JJ. Fatigue characteristics of a co-cured single lap joint subjected to cyclic tensile loads. J Adhes Sci Technol 2002;16(4):347-59.

[6] Shin KC, Lim JO, Lee JJ. The manufacturing process of co-cured single and double lap joints and evaluation of the load bearing capacities of co-cured joints. J Mater Process Technol 2003;138:89-96.

[7] Shin KC, Lee JJ. Bond parameters to improve tensile load bearing capacities of co-cured single and double lap joints with steel and carbon fiber-epoxy composite adherends. J Compos Mater 2003;5:401-20.

[8] Shin KC, Lee JJ. Effect of thermal residual stresses on failure of co-cured lap joints with steel and carbon fiber-epoxy composite adherends under static and fatigue tensile loads. Composites: Part A 2006;37:476-87.

[9] Park SW, Kim HS, Lee DG. Optimum design of the co-cured double lap joint composed of aluminum and carbon epoxy composite. Compos Struct 2006;75:289-97.

[10] Volkersen O. Die Nietkraftverteilung in Zugbeanspruchten mit Konstanten Laschenquerschritten. Luftfahrtforschung 1938;15:41-7.

[11] Williams ML. Stress singularities resulting from various boundary conditions in angular corners of plates in extension. J Appl Mech 1952;19:526-8.

[12] Suo Z. Singularities, interfaces and cracks in dissimilar anisotropic media. Proc Roy Soc Lond, Ser A: Math Phys Sci 1990;427(1873):331-58.

[13] Lekhnitskii SG. Anisotropic plates [Tsai SW, Gordon Cheron T, Trans]. New York: Beach Science Publisher Inc.; 1968.

[14] Tan M. On the singular stress field at the interface of bimaterial systems. PhD thesis, University of Toronto; 1998.

[15] Zuccarello B, Ferrante S. Use of automated photoelasticity to determine stress intensity factors of bimaterial joints. J Strain Anal 2005;40(8):785-800.

[16] Chue CH, Liu CI. Disappearance of free-edge stress singularity in composite laminates. Compos Struct 2002;56:111-29.
[17] Chue $\mathrm{CH}$, Liu CI. A general solution on stress singularities in an anisotropic wedge. Int J Solids Struct 2001;38:6889-906.

[18] Chue $\mathrm{CH}$, Tseng $\mathrm{CH}$, Liu CI. On stress singularities in an anisotropic wedge for various boundary conditions. Compos Struct 2001;54:87-201.

[19] Chue $\mathrm{CH}$, Chen $\mathrm{TH}$, Lee HT. A general solution on stress singularities in the junction of two anisotropic materials. Compos Struct 2002;55:81-93.

[20] Russo A. Giunti Co-cured Fibro Rinforzati a Singola e a Doppia Sovrapposizione: un Metodo di Previsione della Resistenza Basato sui G-SIFs. $\mathrm{PhD}$ thesis, Dipartimento di Meccanica-Università degli Studi di Palermo; 2008.

[21] Lin RL, Ma CC. Antiplane deformations for anisotropic multilayered media by using the coordinate transform method. J Appl Mech 2000;67:597-605.

[22] Penado FE. Singular intensity factors at bimaterial anisotropic interfaces. Compos Struct 2001;52:323-33.

[23] Munskhelishvili NI. Some basic problems of mathematical theory of elasticity. P. Noordhoff and Company; 1953.

[24] Wang CH, Rose LRF. Compact solutions for the corner singularity in bonded lap joints. Int J Adhes Adhes 2000;20:145-54.

[25] Hu N, Wang B, Sekine H, Yao Z, Tan G. Shape-optimum design of a biomaterial single-lap joint. Compos Struct 1998;41:315-30.

[26] Xu JQ Liu YH, Wang XG. Numerical methods for the determination of multiple stress singularities and related stress intensity coefficients. Eng Fract Mech 1999;63:775-90.

[27] Adams RD, Wake WC. Structural adhesive joints in engineering. Elsevier Applied Science Publishers; 1984.

[28] Matthews FL. Joining fibre-reinforced plastics. Elsevier Applied Science Publishers; 1987.

[29] Johnson WS. Adhesively bonded joints: testing, analysis and design. ASTM special technical publication; 1988.

[30] Tong L, Soutis C. Recent advances in structural joints and repairs for composite materials. Kluwer Academic Publishers; 2003.

[31] Belingardi G, Goglio L, Tardini A. Investigating the effect of spew and chamfer size on the stresses in metal-plastics adhesive joints. Int J Adhes Adhes 2002;22:273-82

[32] Hart-Smith LJ. Design and analysis of adhesively bonded joints in fibrous composites and metallic structures. CRS-ACS Ltd; 1998.

[33] Carpinteri A. Stress-singularity and generalized fracture toughness at the vertex of re-entrant corners. Eng Fract Mech 1987;26:143-55.

[34] Broek D. Elementary engineering fracture mechanics. Dordrecht, The Netherlands: Martinhus Nijhoff; 1987.

[35] Broek D. Practical use of fracture mechanics. Dordrecht, NL: Kluver Academic Publisher; 1988.

[36] ASTM D3528-96. Standard test method for strength properties of double lap shear adhesive joints by tension loading; 2002.

[37] Suo Z. Failure of brittle adhesive joints. Appl Mech Rev 1990;43(Suppl. 5):S276-9.

[38] Sutton MA, Deng X, Ma F, Newman Jr JC, James M. Development and application of a crack tip opening displacement-based mixed mode fracture criterion. Int J Solids Struct 2000;37:3591-618.

[39] He MY, Hutchinson JW. Kinking of a crack out of an interface. J Appl Mech 1989;56:270-8.

[40] Wang X, Agrawal CM. Interfacial fracture toughness of tissue-biomaterial systems. J Biomed Mater Res Appl Biomater 1997;38:1-10.

[41] Goland M, Reissner E. The stresses in cemented joints. J Appl Mech 1944;11:A17-27.

[42] Akisanya AR, Fleck NA. Interfacial cracking from the free-edge of a long bimaterial strip. Int J Solid Struct 1997;34,13:1645-65.

[43] Liu D, Fleck NA. Scale effects in the initiation of cracking of a scarf joint. Int J Fract 1998;95:67-88.

[44] Bogy DB. Two edge-bonded elastic wedges of different materials and wedge angles under surface tractions. J Appl Mech 1971;38:377-86.

[45] Reedy Jr ED. Asymptotic interface-corner solutions for Butt tensile joint. Int J Solid Struct 1993;30:767-77. 